\title{
Episodix: a serious game to detect cognitive impairment in senior adults. A psychometric study
}

\author{
Sonia Valladares-Rodriguez ${ }^{\text {Corresp., }}{ }^{1}$ ， Manuel J Fernández-Iglesias ${ }^{1}$ ， Luis Anido-Rifón ${ }^{1}$ ， David Facal ${ }^{2}$, Roberto \\ Pérez-Rodríguez ${ }^{1}$ \\ 1 Department of Telematics Engineering, Universidad de Vigo, Vigo, Spain \\ 2 Department of Developmental Psychology, Universidad de Santiago de Compostela, Santiago de Compostela, Spain \\ Corresponding Author: Sonia Valladares-Rodriguez \\ Email address: soniavr@det.uvigo.es
}

Introduction. Assessment of episodic memory is traditionally used to evaluate potential cognitive impairments in senior adults. The present article discusses the capabilities of Episodix, a game to assess the aforementioned cognitive area, is a valid tool to discriminate among mild cognitive impairment ( $\mathrm{MCl})$, Alzheimer's disease (AD) and healthy individuals (HC), that is, it studies the game's psychometric validity study to assess cognitive impairment.

Materials and methods. After a preliminary study, a new pilot study, statistically significant for the Galician population, was carried out from a cross-sectional sample of senior adults as target users. A total of 64 individuals (28 HC, $16 \mathrm{MCl}, 20 \mathrm{AD}$ ) completed the experiment from an initial sample of 74 .

Participants were administered a collection of classical pen-and-paper tests and interacted with the games developed. A total of six Machine learning classification techniques were applied and four relevant performance metrics were computed to assess the classification power of the tool according to participants' cognitive status.

Results. According to the classification performance metrics computed, the best classification result is obtained using the Extra Trees Classifier ( $F 1=0.97$ and Cohen's kappa coefficient=0.97). Precision and recall values are also high, above 0.9 for all cognitive groups. Moreover, according to the standard interpretation of Cohen's kappa index, classification is almost perfect (i.e., $0.81-1.00$ ) for the complete dataset for all algorithms.

Limitations. Weaknesses (e.g., accessibility, sample size or speed of stimuli) detected during the preliminary study were addressed and solved. Nevertheless, additional research is needed to improve the resolution of the game for the identification of specific cognitive impairments, as well as to achieve a complete validation of the psychometric properties of the digital game.

Conclusion. Promising results obtained about psychometric validity of Episodix, represent a relevant step ahead towards the introduction of serious games and machine learning in regular clinical practice for detecting $\mathrm{MCl}$ or $\mathrm{AD}$. However, more research is needed to explore the introduction of item response theory in this game and to obtain the required normative data for clinical validity. 


\title{
1 Episodix: a serious game to detect cognitive impairment in senior adults. A psychometric study
}

\author{
Sonia Valladares-Rodriguez ${ }^{\mathrm{a}^{*}}$, Manuel J. Fernández-Iglesias ${ }^{\mathrm{a}}$, Luis Anido-Rifón ${ }^{\mathrm{a}}$, David \\ Facal $^{\mathrm{b}}$ and Roberto Pérez-Rodríguez ${ }^{\mathrm{a}}$
}

\begin{abstract}
Introduction. Assessment of episodic memory is traditionally used to evaluate potential cognitive impairments in senior adults. The present article discusses the capabilities of Episodix, a game to assess the aforementioned cognitive area, is a valid tool to discriminate among mild cognitive impairment (MCI), Alzheimer's disease (AD) and healthy individuals (HC), that is, it studies the game's psychometric validity study to assess cognitive impairment.
\end{abstract}

Materials and methods. After a preliminary study, a new pilot study, statistically significant for the Galician population, was carried out from a cross-sectional sample of senior adults as target users. A total of 64 individuals (28 HC, $16 \mathrm{MCI}, 20 \mathrm{AD})$ completed the experiment from an initial sample of 74. Participants were administered a collection of classical pen-and-paper tests and interacted with the games developed. A total of six Machine learning classification techniques were applied and four relevant performance metrics were computed to assess the classification power of the tool according to participants' cognitive status.

Results. According to the classification performance metrics computed, the best classification result is obtained using the Extra Trees Classifier (F1=0.97 and Cohen's kappa coefficient=0.97). Precision and recall values are also high, above 0.9 for all cognitive groups. Moreover, according to the standard interpretation of Cohen's kappa index, classification is almost perfect (i.e., 0.81-1.00) for the complete dataset for all algorithms.

Limitations. Weaknesses (e.g., accessibility, sample size or speed of stimuli) detected during the preliminary study were addressed and solved. Nevertheless, additional research is needed to improve the resolution of the game for the identification of specific cognitive impairments, as well as to achieve a complete validation of the psychometric properties of the digital game.

Conclusion. Promising results obtained about psychometric validity of Episodix, represent a relevant step ahead towards the introduction of serious games and machine learning in regular clinical practice for detecting MCI or AD. However, more research is needed to explore the introduction of item response theory in this game and to obtain the required normative data for clinical validity.

\section{INTRODUCTION}

People living with dementia around the world is steadily increasing, because the primary risk factor is old age (Winblad et al., 2016). Their number almost double every 20 years, and will reach 131.5 million by 2050 (Prince, 2015). Alzheimer's disease (AD) is the most common cause of dementia, as it accounts for an estimated $60 \%$ to $80 \%$ of cases diagnosed. It is considered a slowly progressive brain disease that begins well before clinical symptoms

${ }^{*}$ Corresponding author. Tel.: +34-986-814073

E-mail address: soniavr@det.uvigo.es. 
emerge. Due to this, AD is among the most prevalent medical conditions and one of the most relevant health threats worldwide (WHO \& Others, 2015).

Diagnosing AD requires a careful and comprehensive health evaluation, including an examination of the patient's mental and functional status, such as, memory, language, visuospatial abilities, executive functions, or living diary activities, among others. Diagnostic procedures for AD are based on clinical procedures, including neuropsychological pen-and-paper tests (Lezak, 2004; Howieson \& Lezak, 2010). These tools are used to perform a cognitive evaluation that may be general or specific to certain cognitive areas (e.g., memory, language, attention, visuospatial capabilities, etc.).

However, classical tests have some relevant limitations, such as being perceived as intrusive (Chaytor \& SchmitterEdgecombe, 2003); being influenced by the white-coat effect (Mario et al., 2009); providing a late diagnosis (Holtzman, Morris \& Goate, 2011); lacking ecological validity (Farias et al., 2003; Knight \& Titov, 2009); being strongly dependent on confounding factors (e.g., age, educational level(Cordell et al., 2013), practice effect (Hawkins, Dean \& Pearlson, 2004; Lezak, 2004)), or being prone to processing errors due to their manual processing.

Due to the need of alternate ecological mechanisms supporting an early diagnosis of cognitive impairment, the scientific literature discusses several approaches such as the digitalization of classical tests (Robinson \& Brewer, 2016), the introduction of game-inspired design approaches (e.g., rewards, challenges, simulated environments) (Tong \& Chignell, 2014) and the implementation of technology-based solutions (e.g., immersive 3D environments, virtual reality, online interactive software applications) (Parsons et al., 2004; F. et al., 2010; L. et al., 2010; Plancher et al., 2012; Nolin et al., 2013, 2016; Nori et al., 2015; Iriarte et al., 2016), among others. The proposal discussed in this paper relies on the use of gamification techniques, machine learning and the introduction of digital touch devices. More specifically, this paper discusses a psychometric study about a digital game - named Episodix - a video game to assess episodic memory, based on the gamification of the California Verbal Learning Test (CVLT).

The design procedure and a preliminary validation of Episodix was discussed in a previous work (ValladaresRodriguez et al., 2017). Promising results were obtained concerning both Episodix's prediction capabilities and acceptability by target users. Namely, this tool provided a promising accurate discrimination between healthy participants and individuals suffering mild cognitive impairment, while being highly accepted by target users. Moreover, cognitive areas tackled (Lezak, 2004), during game evaluation process, are episodic memory, semantic memory and procedural memory. These cognitive areas, and specially the first one, are early markers of cognitive alterations with a relevant diagnostic value for mild cognitive impairment (MCI) and AD (Juncos-Rabadán et al., 2012; Facal, Guàrdia-Olmos \& Juncos-Rabadán, 2015). However, additional research is needed to achieve a complete psychometric validation, and also, to improve the predictive ability to detect specific cognitive impairment conditions.

Besides, after a thorough revision of the scientific literature in this field (Helkala et al., 1989; Perry, Watson \& Hodges, 2000; Whitwell et al., 2007; Werner et al., 2009; Plancher et al., 2010; Grambaite et al., 2011; Libon et al., 2011; Raspelli et al., 2011; Aalbers et al., 2013; Tarnanas et al., 2013; Nolin et al., 2013; Fukui et al., 2015; Kawahara et al., 2015), included in our previous methodological review (Valladares-Rodriguez et al., 2016), it became apparent that more research was needed to obtain a robust psychometric validation that would eventually support the introduction of cognitive games such as Episodix, as a diagnostic tool in everyday clinical practice. In other words, there is a need to empirically evaluate the claim that serious games are a valid means to assess constructs and behaviors with at least the same validity than traditional approaches (Kato \& de Klerk, 2017).

Thus, additional research was targeted to provide an answer to the following research question: is the Episodix game a valid tool - with a statistical significance for older adults - to discriminate between MCI, AD and healthy individuals? For this, a psychometric validity study of the aforementioned tool was carried out, thorough a pilot study with a statistically significant sample. On the one hand, the psychometric validity analysis was performed according to the recommendations from the Standards for Educational and Psychological Testing (AERA, APA \& NCME, 2014). For this, we focused on predictive validity, that is, on how well Episodix predicts cognitive impairment, overcoming the limitations encountered during the preliminary study discussed above (Valladares-Rodriguez et al., 2017). Innovative machine learning techniques were applied, which were introduced to support medical prediction and classification related to cancer or heart diseases (Lehmann et al., 2007; Maroco et al., 2011). The application of this approach is relatively new in cognitive ageing or cognitive impairment studies. A k-fold cross-validation strategy was applied for training and testing the classification models.

The rest of the paper is organized as follows: Sect. 2 introduces the materials and methods utilized in this pilot study, namely test participants' characterization and enrollment, mathematical apparatus used and data analysis performed; Sect. 3 discusses the outcomes of a pilot experience involving a cross-sectional study participating 74 
94

95

96

97

98

99

100

101

102

103

104

105

106

107

108

109

110

111

112

113

114

115

116

117

118

119

120

121

122

123

124

125

126

127

128

129

130

131

132

133

134

135

136

137

138

139

140

141

142 senior adults; Sect. 4 discusses the psychometric validation process, and finally the main conclusions of this work are summarized.

\section{MATERIALS and METHODS}

\subsection{Study design}

\subsubsection{Sample description}

This study was carried from a cross-sectional sample of senior adults as target users (cf. Fig 1). Initially the sample was composed of 74 people over 55 years old (range=57-95; average=77.03; SD=7.23), from them 47 women and 27 men (average $=34 \%$ males; SD: 0.48), with an average of 6.90 years of education completed. Even though usual onset age of AD is 65 years old (Albert et al., 2011), according to several studies (Chetelat et al., 2005; Rasquin et al., 2005; Juncos-Rabadán et al., 2014) we set the inclusion age in our study at 55 years in order to assess mild cognitive impairment (MCI), an early cognitive damage stage.

All participants were recruited in the Pontevedra province (Galicia, Spain) at the nursing home of Santa Marta in Vigo, at the Association of Relatives of Alzheimer's Patients of Morrazo (AFAMO), and finally, at the Association of Relatives of Alzheimer's Patients and other Dementias of Galicia (AFAGA). The basic inclusion criterion was being 55+ years old, and exclusion criteria included an advanced cognitive impairment, severe motor, hearing or visual disability, and explicit rejection of technology — all issues validated by professionals of the aforementioned associations. Participants should attend a socio-cognitive workshop in any of the associations indicated above, and no previous educational level or technological skills were required.

During the pilot study, four people were dismissed due to advanced AD; one person passed away; one individual quitted compulsory the socio-cognitive workshop before its completion, and finally 4 people voluntarily quitted the pilot study. Therefore, a cross-sectional sample of 64 subjects eventually completed the study, from them 20 subjects with $\mathrm{AD}, 16$ participants with MCI, and finally, 28 as controls - referred to as the HC group in the rest of this paper-.

The study design was approved by the institutional ethics committee (i.e., Galician Ethics Committee for research (Spain), number code 2016/477) and was conducted in accordance with the provisions of the Declaration of Helsinki, as revised in Seoul 2008. Before their involvement in the pilot project, all participants read and understood patient information sheets and gave their written informed consent to use the anonymized data gathered.

Finally, the pilot study was organized in four sessions per subject, lasting 45 minutes each, within a one-month interval. More specifically, three of the sessions were dedicated to game interaction and the last one to classical cognitive evaluation, as discussed below.

\subsubsection{Cognitive assessment}

Participants were administered a collection of classical pen-and-paper tests and a questionnaire about sociodemographic aspects such as exercise practice, socialization or present medications to gain some insight on the quality of life of participants and their cognition levels (Banerjee et al., 2006).

With respect to the above mentioned tests, MMSE - Mini-metal Examination State (Cockrell \& Folstein, 1987), the Spanish version of CVLT - California Verbal Learning Test, AD8 - Adapted Dementia Screening Interview , ADL - Barthel scale of activities of daily living, and a questionnaire about memory complaints were administered (cf. Table 1). Data gathered using these tests were used as golden standard data and also to initially discriminate subjects suffering MCI from healthy controls. According to standard diagnostic criteria (Petersen, 2004; Albert et al., 2011; JuncosRabadán et al., 2012; Campos-Magdaleno et al., 2017), MCI would likely be present for participants that would present normal cognitive functions and their capabilities to carry out everyday activities are not compromised, they would refer memory complaints, a low score in the Spanish version of CVLT according to subject's age and educational background, and a level equal or greater than 2 in the AD8 test. Participants who suffered AD -facilitated by participating organizations with a prior medical diagnosis- were not asked to take the Spanish version of CVLT to avoid frustration. 
Finally, demographic and lifestyle information collected (cf. Fig 1) shows that social interaction and physical activities decrease with increasing cognitive impairment. Besides, participating individuals are in most cases subject to chronic medication due to age-related conditions such as hypertension, hypercholesterolemia, or diabetes, and medication increases with age and increasing cognitive impairment.

\footnotetext{
Legend: Bars are distributed by cognitive groups: A. gender: men/women; B. age; C. chronic treatment: yes/no (i.e., 1/0); D. educational level, E. exercise level and F. socialize level, all of them based on a 5-point Likert scale: 1(never) to 5 (always).
}

Fig 1. Main general subjects' characteristics according to cognitive group (i.e., $\mathrm{HC}, \mathrm{MCI}$ and $\mathrm{AD}$ ).

Table 1. Comparative of participants' cognitive assessment by cognitive group

Note: MMSE cut-off score: 9-12 means AD is likely present. Spanish version of CVLT cut-off scores computed according to the results of Total Trial 1-5, Short Delay Free Recall, Long Delay Free Recall, age and years of education (Albert et al., 2011; Juncos-Rabadán et al., 2012; Campos-Magdaleno et al., 2017). Memory complaints (by participants and informants) based on a 5-point Likert scale: 1(never forget) to 5 (always forget). AD8, 0-1: normal cognition; 2 or greater: cognitive impairment is likely to be present. Finally, ADL: 0-20: total dependency; $21-$ 60: severe dependency; 61-90: moderate dependency; 91-99: small dependency; and 100: autonomy.

\subsubsection{Serious game assessment}

Gaming sessions consisted on three consecutive phases. Firstly, participants would play the first part of Episodix (cf. A and B in Fig 2), which target immediate and short-term memory. Then, they would play two additional games -to assess semantic and procedural memory (cf. C and D in Fig 2 and Appendix C with supplementary information)-, and finally participants would play the second part of Episodix to assess long-term memory and yes/no recognition capabilities. Note that this approach emulates the administration of CVLT, only that using serious games instead of pen-and-paper tests, and breaks being used to evaluate other cognitive markers.

From a technological point of view, games were developed in Unity (Unity, 2016) and were run in a Samsung Galaxy Note Pro (SM-P900) tablet device. Thus, senior adults interacted with a touch version of the tool to enhance its usability. Episodix also offers multi-language support, and all stimuli are additionally presented in textual and audio formats to enhance accessibility.

Finally, serious game assessment was carried out at the same time and location as the compulsory socio-cognitive workshops to guarantee the ecological validity of neuropsychological evaluation by integrating the latter in participating participants' daily routines. This was particularly relevant for participants with cognitive impairment, for whom there is a high prevalence of the white-coat effect (Mario et al., 2009).

\subsection{Data analysis}

\subsubsection{Statistical significance}

The sample of senior adults recruited fulfills the statistical representativeness criteria for the senior population in Galicia, Spain. The sample size was computed according to the minimum sample for a prevalence study based on proportional stratified sampling (Barlett, Kotrlik \& Higgins, 2001):

$$
n=\frac{N * Z \alpha^{2} * p * q}{\varepsilon^{2} *(N-1)+Z \alpha^{2} * p * q}
$$


where $\mathrm{N}$ represents the size of the Galician population over 55 years according to the National Statistics Institute; $\mathrm{Z}$ is the statistical confidence level (i.e., $1.96 \approx 95 \%$ ); $\mathrm{p}$ represents the probability of AD prevalence in Galicia according to the National Statistics Institute; $q$ is the probability of AD's absence (i.e., $q=1-p$ ); indicates admissible error (i.e., 7.5\%), which is within the usual values in research studies (i.e., $5 \%-10 \%$ ). As a result, a sample of $n=62$ individuals would be required for the results obtained during the pilot study to be statistically significant.

\subsubsection{Psychometric study}

To assess whether Episodix is a valid cognitive game to discriminate between individuals suffering MCI, individuals suffering AD or participants not suffering cognitive impairment, we focused on psychometric criterion validity (Souza, Alexandre \& Guirardello, 2017), in particular predictive ability. In other words, we tested the validity of serious games by comparing them with golden standard criteria (i.e., classical tests described in Sect. 2.1.2).

To do that, recent machine learning classification techniques were applied. Particularly, we selected popular techniques in medical research based on supervised learning (Lehmann et al., 2007; Tripoliti et al., 2010; Maroco et al., 2011), in order to obtain a prediction or classification score about cognitive impairment (i.e., MCI, AD or absence of cognitive problems). Techniques introduced in the preliminary study were also applied now (i.e., LR, SVM and RF) (Valladares-Rodriguez et al., 2017), and three new techniques were introduced (i.e., ET, AB and GB) due to their high efficacy when applied to classification problems.

- LR - Logistic regression. It is a statistical method for analyzing a dataset in which there are one or more independent variables that determine an outcome. The outcome is measured with a dichotomous variable (in which there are only two possible outcomes). This is a simple technique widely used in predictive analysis in the medical field.

- SVM - Support Vector Machines (Hearst et al., 1998). This technique is widely used for clustering and classification. It aims at building a model able to predict to which category a new element added to the model would belong. SVM represents each element as a point in an n-dimensional space. Then, different element classes correspond to clusters in that space. Separating hyperplanes, named "support vectors", are computed to discriminate among clusters.

- RF - Random Forests (Breiman, 2001). This algorithm, also known as random decision forests, is a perturb-andcombine technique specifically designed for decision trees where random alterations are introduced in the learning process. RF is an ensemble learning method that provides better prediction capabilities tan other decision tree-based methods. Besides, it corrects the latter's' trend to overfitting.

- ET - Extra Trees Classifier (Geurts, Ernst \& Wehenkel, 2006) is based on unpruned top-down decision trees and are also based on the perturb-and-combine strategy. They are typically used to enhance precision in predictions provided by traditional decision trees. "Extra" stands for extremely randomized trees.

- GB - Gradient Boosting Classifier (Payan \& Montana, 2015). Technique, based on decision trees, which produces a prediction model in the form of an ensemble of weak prediction models. Namely, it optimizes a cost function over function space by iteratively choosing a function (weak hypothesis) that points in the negative gradient direction.

- AB - Ada Boost Classifier (Freund \& Schapire, 1997). This machine learning meta-algorithm, is adaptive in the sense that subsequent weak learners are tweaked in favor of those instances misclassified by previous classifiers. Moreover, this algorithm is sensitive to noisy data and outliers.

For each of the previous algorithms, quality metrics widely used in medical studies were computed:

- F1-score: weighted harmonic average of precision and recall.

$$
F 1(F 1 \text { score })=2 * \frac{P * R}{P+R}
$$

- Cohen's kappa coefficient (Cohen, 1960) provides a measure of the validity of the classification when confronted with a random result. $k$ value ranges are interpreted according to the existing consensus (Landis \& Koch, 1977): 0-0.20 Insignificant (i.e., ML-based classification would not be distinguishable from random classification); 0.210.40 Median; 0.41-0.60 Moderate; 0.61-0.80 Substantial; and 0.81-1.00 Almost perfect; 


$$
k(\text { Cohen's kappa })=1-\frac{(1-P o)}{(1-P e)}
$$

Where $P_{o}$ is the relative observed agreement (i.e., accuracy), and $P_{e}$ the estimated or hypothetical probability of random agreement.

- Recall or sensitivity: game's ability to identify actually impaired people to avoid false negatives.

$$
\text { Recall }=\frac{T P}{(T P+F N)}
$$

- Precision or ratio of relevant information over total information obtained during the prediction process.

$$
\text { Precison }=\frac{T P}{(T P+F P)}
$$

About the last two metrics, we computed the precision-recall curve as an indication of classification quality. Note that a larger area below the curve indicates better precision (i.e., game's ability to obtain positive predicted values) and better recall (i.e., game's ability to avoid false negatives). This curve is commonly used in the analysis of information retrieval systems as an alternative to the ROC curve because it provides a more informative picture of the classifier's performance (Davis \& Goadrich, 2006).

The aforementioned metrics were reported as macro values, because they are more convenient to show the performance of the classifiers with data sets that are not evenly distributed over the available categories. The calculation of macro metrics is based simply on computing the metrics for each label or class and then obtaining its un-weighted average.

Finally, to prevent over-fitting and artificial classification metrics due to the use of the same data for training and testing of classifiers, a k-fold cross-validation strategy was adopted to train and evaluate the 4 proposed classifiers. Furthermore, the machine learning library, Scikit-Learn (Pedregosa et al., 2011), was used for all data analytics, under a Python ecosystem. Missing data due to the different difficulty levels achieved by different participants, was addressed by means of substitution by cognitive group (i.e., AD, MCI or HC). This frequently used method replaces the missing data for a given attribute by the mean of all known values of that attribute, for the different cognitive groups (Batista \& Monard, 2003; Garc’’ia-Laencina, Sancho-Gómez \& Figueiras-Vidal, 2010).

\section{RESULTS}

This section presents the results of the psychometric study of Episodix. Both aspects were analyzed for the complete sample $(n=64)$ and for the cognitive groups defined HC $(n=28), M C I(n=16)$ and $\operatorname{AD}(n=20)$.

\subsection{General and cognitive characteristics of participants}

Participants (cf. Fig 2) were characterized according to the parameters below (characteristics are expressed as average values and their standard deviation).

Regarding general demographic details (cf. Fig 1) for each cognitive group:

- Age: participants have an average of $75.57 \pm 7.14$ years old for controls; $76.87 \pm 9.33$ years old for participants with MCI; and, finally, $79.15 \pm 4.91$ years old for people with AD;

- Gender: the average of females in the sample was $0.14 \pm 0.35$ for HC group; $0.37 \pm 0.50$ for females with MCI; and $0.60 \pm 0.50$ for $\mathrm{AD}$ group.

- Chronic treatment (i.e., a dichotomous variable "yes" or "no"): the $0.82 \pm 0.39$ for healthy participants; the $0.81 \pm 0.40$ for people with $\mathrm{MCI}$; and a mean of $0.90 \pm 0.31 \mathrm{AD}$ patients under treatment.

- Educational level (i.e., years of education): $1.34 \pm 0.82$ for people without cognitive impairment; $1.78 \pm 0.41$ for

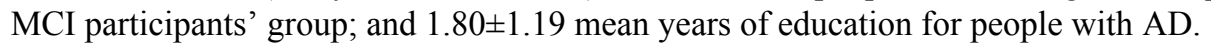


- Exercise level (i.e., 5-point Likert scale: 1(nothing) to 5 (a lot)): $3.82 \pm 1.09$ for controls; $3.75 \pm 0.86$ for MCI participants' group; and 3.30 \pm 0.92 for AD group.

- Socialize level (i.e., 5-point Likert scale: 1(nothing) to 5 (a lot)): $3.58 \pm 1.10$ for HC participants; $3.37 \pm 0.88$ for participants with MCI; and 2.80 \pm 0.95 for people affected by AD.

With respect to the outcomes of classical testing (cf. Table 1), once again according to each cognitive group:

- MMSE: $26.93 \pm 2.11$ for HC participants; $25.37 \pm 2.55$ for people with MCI; and $23.00 \pm 4.38$ for people affected by $\mathrm{AD}$, over a total score of 30 .

- Spanish version of CVLT, according to standard diagnosis criteria. Total Trial 1-5: 47.25 \pm 5.83 [HC group] and 34.62 \pm 9.50 [MCI group]; Short Delay Free Recall: 9.93 \pm 2.55 [HC group] and 6.31 \pm 1.74 [MCI group]; and finally, Long Delay Free Recall: 10.93 \pm 2.39 [HC group] and 5.87 \pm 3.07 [MCI group].

- AD8: in this case the average score for $\mathrm{HC}$ was $1.39 \pm 1.47 ; 1.75 \pm 1.18$ for participants with $\mathrm{MCI} ; 5.40 \pm 1.31$ for AD participants.

- ADL Barthel scale: 102.07 \pm 6.39 for HC individuals; $100 \pm 7.28$ for MCI participants; and finally, $91.85 \pm 11.03$ for participants affected by AD.

- Subject memory complaints raised by participants about themselves: $1.99 \pm 0.51$ for HC; $1.99 \pm 0.57$ for participants with MCI; and 2.47 \pm 0.50 for AD participants.

- Subject memory complaints raised by an informant: $1.82 \pm 0.73$ for HC participants; $1.78 \pm 0.41$ for MCI people; $2.46 \pm 0.64$ for people affected by AD.

Legend: A and B: Episodix, C: semantic memory game and D: procedural memory game. Photo permission was required and granted in the patient informant sheet. Photo credit: Sonia Valladares-Rodriguez.

Fig 2. Some captures of cognitive game sessions.

\subsection{Psychometric study outcomes}

All participants played two complete game sessions, each session consisting of two Episodix phases separated by semantic \& procedural memory games during the break between the two Episodix phases. Obtained datasets were processed with the machine learning algorithms enumerated above, that is, Extra Trees classifier (ET); Gradient Boosting Classifier (GB); Ada Boost Classifier (AB); Support Vector Machines (SVM); Logistic Regression (LR); and Random Forest (RF).

Firstly, ML algorithms were applied to the dataset composed of Episodix data, focusing on psychometric predicted validity. Thus, a total of 191 triplets were included in the dataset. The metrics below were computed to estimate the classification abilities (cf. Table 2, sorted according to decreasing $\mathrm{k} \downarrow$ ):

- F1 score: average values obtained were 0.92 for GB; 0.91 for ET; 0.89 for ET; 0.81 for AB; 0.80 for SVM; and 0.76 for LR.

- Cohen's kappa: the average value obtained for the concordance of the observed participants' classification for each algorithm was: 0.89 for GB; 0.91 or ET; 0.86 for RF; 0.76 for AB; 0.75 for SVM; and 0.70 for LR.

- Recall was computed for each cognitive group:

O ET: 0.96 for $\mathrm{HC}, 0.92$ for MCI, and 0.91 for participants with AD.

$\mathrm{OGB}$ : in the range of $0.91-0.95$.

$\mathrm{AB}$ : in the range of $0.76-0.96$.

SVM: in the range of $0.75-0.93$.

O LR: 0.74 for $\mathrm{HC}, 0.73$ for MCI, and 0.94 for participants with AD.

$\mathrm{ORF}$ : in the range of $0.86-1$.

- Precision, again by cognitive group:

$\mathrm{HC}$ : in the range of $0.91-0.99$. 
MCI: in the range of $0.42-0.87$.

$\mathrm{AD}$ : in the range of $0.91-0.96$.

2. Metrics about psychometic predicted validity of the Episodix game.

Note: Episodix dataset (191 triples). ML algorithms: ET: Extra Trees classifier; GB: Gradient Boosting Classifier; AB: Ada Boost Classifier; SVM: Support Vector Machines; LR: Logistic regression; and RF: Random forest. Metrics: F1 score; Cohen's Kappa (i.e., used as index to order best classification); recall and precision, the last two distributed by cognitive group. Experiments were performances with cv-fold cross validation $(\mathrm{cv}=55)$ and default configuration in ML algorithms.

The same ML algorithms were applied to a dataset including Episodix, semantic memory and procedural memory data (458 triples). The metrics below were computed to estimate the classification abilities (cf. Table 3, again sorted according to decreasing $\mathrm{k} \downarrow$ ):

- F1 score: average values obtained were .97 for ET; 0.97 for RF; 0.96 for GB; 0.91 for SVM; 0.84 for LR; and 0.84 for $\mathrm{AB}$.

- Cohen's kappa: the average value obtained for the concordance of the observed participants' classification for each algorithm was: 0.97 for ET; 0.967 for RF; 0.95 for GB; 0.90 for SVM; 0.81 for LR; and 0.80 for AB.

- Recall by cognitive group:

$\mathrm{HC}$ : in the range of $0.77-0.96$.

MCI: in the range of $0.78-0.99$.

$\mathrm{AD}$ : in the range of $0.98-0.99$.

- Precision, by cognitive group:

$\mathrm{OHC}$ : in the range of $0.90-0.99$.

MCI: in the range of $0.67-0.93$.

$\mathrm{AD}$ : in the range of $0.93-1.00$.

Table 3. Metrics about psychometric predicted validity of the Episodix, semantic memory and procedural memory games.

Note: Episodix, semantic memory and procedural memory dataset (458 triples). ML algorithms: GB: Gradient Boosting Classifier; ET: Extra Trees classifier; SVM: Support Vector Machines; LR: Logistic regression; AB: Ada Boost Classifier; and RF: Random forest. Metrics: F1 score; Cohen's Kappa (i.e., used as index to order best classification); recall and precision, the last two distributed by cognitive group. Experiments were performances with cv-fold cross validation $(\mathrm{cv}=55)$ and default configuration in ML algorithms.

As higher values were obtained for the metrics computed in this case, the precision-recall curve was also computed for the complete dataset including Episodix, semantic memory and procedural memory data. The curve depicts the average of precision and recall, together with the area below the curve, for each cognitive group. In this case, as Fig 3 shows, a high area under the curve is obtained for all ML algorithms, specially, for GB, RF and ET with an average precision higher to 0.93. All algorithms show a high precision-recall area for the AD group (i.e., value higher to 0.95), followed by the HC group (i.e., value higher to 0.95 except for AB and LR), and finally the MCI group (i.e., value higher to 0.74, except again for AB and LR).

Legend: ML algorithms: A. [GB: Gradient Boosting Classifier]; B. [AB: Ada Boost Classifier]; C. [ET: Extra Trees classifier]; D. [LR: Logistic regression]; E. [SVM: Support Vector Machines]; and F. [RF: Random forest]. Metrics: precision-recall curve average for each cognitive group. Experiments were performances with cv-fold cross validation $(\mathrm{cv}=55)$.

Fig 3. Comparative of Precision-Recall curve metric to evaluate classifier output 
Furthermore, we computed the collection of the 10 most informative variables for classification for both datasets. For this two models of features selection, based on chi-squared and ANOVA F-value statistics with similar results. As a consequence, the set of most informative features are (cf. Appendix A for additional details):

- $\quad$ EPISODIX dataset: number of failures during immediate recall phase, short-term recall and long-term recall; number of omissions during long-term recall with semantic clues; and number of repetitions during yes/no recognition phase.

- $\quad$ EPISODIX $+\mathrm{S}+\mathrm{P}$ dataset: in general, the most informative variables are the same as in the previous case. However, in this case, time duration of procedural memory tasks is included among the 10 most informative variables.

Finally, we applied again the six ML algorithms with a subset including the aforementioned 10 most informative variables for classification for both datasets (cf. Appendix B for additional details). On the one hand, using only the Episodix data set, quality metrics dropped in general terms. For instance, the F1-score dropped to 0.59-0.87 from an initial range of $0.76-0.93$. In the same line, average precision-recall area was reduced (i.e., from 0.89-0.72 to 0.540.8). On the other hand, if we consider the metrics obtained for the three games using the most informative features, they also dropped slightly. By focusing on the best performance obtained using the Gradient Boosting classifier, F1 changes from 0.96 to 0.93 ; Cohen's kappa raises from 0.94 to 0.95 ; and finally, precision-recall area remains the same (i.e., 0.93 for ANOVA F-value feature selection). Fig 4 below depicts the comparative of Precision-Recall curve metrics using the Gradient Boosting classifier. The area is larger using all the features (i.e., 81 features for Episodix and 100 features for Episodix $+\mathrm{S}+\mathrm{P}$ ) in comparison to the 10 most informative variables, including subject's age and genre. Furthermore, in the case of ANOVA F-value selection, general results are equal, showing a small area in the prediction of the MCI group (i.e., it varies from 0.90 to 0.86 ).

Legend: A. [Episodix data set, GB: Gradient Boosting Classifier and chi-squared feature selection]; B. [Episodix data set, GB: Gradient Boosting Classifier and ANOVA F-test feature selection]; C. [Episodix data set, GB: Gradient Boosting Classifier and all features]; D. [Episodix + S $+P$ data set, GB: Gradient Boosting Classifier and chi-squared feature selection]; E. [Episodix+S+P data set, GB: Gradient Boosting Classifier and ANOVA F-test feature selection]; and F. [Episodix+S+P data set, GB: Gradient Boosting Classifier and all features].

Fig 4. Comparative of Precision-Recall curve metric using the best performance algorithm (i.e. Gradient Boosting classifier).

\section{DISCUSSION}

This article discusses the psychometric study of Episodix, a game-based battery to evaluate cognitive impairment. A previous research stated the preliminary prediction ability of this instrument (Valladares-Rodriguez et al., 2017). The justification of this new research is to overcome the limitations detected in that previous study, specially, those related to the representativeness of the population sample. This, in this case the objective would be to validate from a sound psychometric perspective, the diagnostic abilities of the game supported by supervised machine learning algorithms.

In this new study, a total of 64 individuals completed the pilot experiment from an initial sample of 74. Twentyeight participants were included in the healthy control group, while 16 people presented MCI and 20 suffered AD. The sample was statistically representative of the size of Galician senior population, which in turn shares demographic characteristics with the population in many other rural areas in Europe. Besides, the sample is also representative from a research perspective (Barlett, Kotrlik \& Higgins, 2001). Particularly, according to recommendations for this type of studies (Hajian-Tilaki, 2014), the sample utilized satisfies the required total sample size (i.e., $41<$ number of participants $<78$ ) for estimating a specificity between $0.90-0.95$, for a marginal error of 0.07 and prevalence of 0.1 .

On the other side, participating senior adults felt comfortable during the evaluation sessions embedded in their daily routines, which makes this tool an innovative ecological mechanism supporting an early diagnosis of cognitive impairment. Besides, no technological impediments were detected insofar usability is concerned due to the introduction of touch devices. To sum up, this novel approach to neuropsychological evaluation overcomes the 
434

435

436

437

438

439

440

441

442

443

444

445

446

447

448

449

450

451

452

453

454

455

456

457

458

459

460

461

462

463

464

465

466

467

468

469

470

471

472

473

474

475

476

477

478

479

480

481

482

483

484

485

486

487

limitations of classical tests, as it avoids the white-coat effect, confounding factors or errors common while computing final scores. All of these issues are even more relevant when target users are senior adults.

After a preliminary psychometric study of Episodix (Valladares-Rodriguez et al., 2017) (i.e., content, face and ecological validity), the research discussed in this article was focused on criterion validity, that is, in the ability to identify cognitive impairment with statistical validity. For this, a collection of six ML algorithms widely used in medical research were selected according to their classification performance (Lehmann et al., 2007; Tripoliti et al., 2010; Maroco et al., 2011). More specifically, the ones applied in the mentioned preliminary study were also considered in this new research (i.e., Support Vector Machines (SVM); Logistic regression (LR); and Random forest $(\mathrm{RF})$ ) and three new algorithms were also introduced (i.e., Extra Trees classifier (ET); Gradient Boosting Classifier (GB); AB: Ada Boost Classifier (AB)), all of them run according to the default configuration, which in turn materializes one of the future research lines identified in the preliminary study. Furthermore, this new study introduces an additional improvement related to the training and testing data by introducing a 55 -fold stratified cross validation procedure thanks to the richer dataset available. This mechanism prevents overfitting and artificial classification metrics due to the use of the same data for training and testing of classifiers, and dramatically improves the classical $80 / 20$ data split strategy for training and testing. Moreover, despite of applying mean imputation to handle missing data, which may contribute to underrepresent the variability in the data, the impact in actual data set is limited because mean this value was computed by cognitive group and not for the all subject. As a consequence, variability was respected by cognitive group. Besides, the amount of missing data was low, as it was only caused by different difficulty levels achieved by participants.

Attending to the classification results obtained with the first dataset in this new research including Episodix only, better results are obtained for $\mathrm{GB}$, with an average value of $\mathrm{F} 1=0.93$ and a concordance Cohen's kappa $=0.90$, over a maximum of 1. Besides, the recall and precision values obtained are all of them above 0.94 , except precision for the MCI group (i.e., 0.79). These results demonstrate that Episodix has the ability to predict mild cognitive impairment, according both to the values computed for the harmonic average of precision and sensitivity (i.e., F1), and to the concordance of results when considering a random classification (i.e., Cohen's kappa). Results are also promising for $\mathrm{ET}$ and RF, followed by $\mathrm{AB}$ and SVM in decreasing order of classification quality. Worst results were obtained for LR, particularly precision for participants with MCI, as summarized in Table 2.

When applying the above ML algorithms to the second dataset (i.e., Episodix, semantic and procedural memory games), it can be observed that all metrics slightly improve. In this case, best results are obtained with the ET algorithm with F1=0.97 and Cohen's kappa $=0.94$. Precision and recall values are also high, above 0.9 for all cognitive groups. With respect to the other ML algorithms, the next one would be ET, followed by RF, AB, SVM, and finally LR with slightly better results when compared to the ones obtained with the first dataset.

Note that results are sorted according to the Cohen's kappa index because it provides a measure of the validity of the classification when compared to a random result. Moreover, according to the standard interpretation of this index (Cohen, 1960), classification is almost perfect (0.81-1.00) for the complete dataset for all algorithms, except AB, SVM and LR in the case of the Episodix dataset, which can be considered as substantial (0.61-0.80).

Furthermore, we computed precision-recall curves as a useful measure of success of prediction when the classes are imbalanced. We plotted them as a multi-category class including average area and disaggregated by cognitive impairment (i.e., $\mathrm{HC}, \mathrm{MCI}$ and $\mathrm{AD}$ groups). In information retrieval, precision is a measure of result relevancy, while recall is a measure of how many truly relevant results are returned. In other words, a high area under the curve represents both high recall and high precision in the prediction of cognitive impairment (i.e., participants affected by $\mathrm{MCI}$ or $\mathrm{AD}$ ), where high precision relates to a low false positive rate, and high recall relates to a low false negative rate. Thus, as can be observed in Fig 3, high scores are for ML classifiers based on ensemble methods (e.g., RF, GB, and ET, where precision-recall area is higher to 0.90 for all types of participants- $\mathrm{HC}, \mathrm{MCI}$ or $\mathrm{AD}$ groups- ). Thus, according to these outcomes and the statistical representativeness of the sample in Galicia, all of them allow to predict or discriminate cognitive impairment with high accuracy.

Finally, regarding the most informative features for classification, both using chi-squared and ANOVA F-value statistics methods, results were similar, these being the number of failures during immediate recall phase, short-term recall and long-term recall; number of omissions during long-term recall with semantic clues; number of repetitions during yes/no recognition phase; and time duration of procedural task. Particularly, we computed again ML metrics using the reduced dataset. In general terms, the precision-recall area is higher using all the features (i.e., 81 features for Episodix and 100 features for Episodix $+\mathrm{S}+\mathrm{P}$ ) in comparison with the 10 most informative, including subject's age and genre. However, in the case of the ANOVA F-value selection and GB classifier, general results are equal, showing a slight decline in the prediction of MCI group (i.e., it varies from 0.90 to 0.86 ), but a better prediction in the case of 
$\mathrm{HC}$ and $\mathrm{AD}$ participants. These findings, using an $80 \%$ smaller dataset, have a future potential for a commercial product, where most likely computational restrictions would be a key issue. In the present pilot study, runtime of all experiments was the order of milliseconds.

To conclude, limitations detected during the preliminary study (Valladares-Rodriguez et al., 2017), were conveniently addressed and solved. Particularly, with respect to accessibility, senior adults clearly preferred a touch interface better than traditional keyboard and mouse, and this was included in the new experiment. Other relevant aspect was the limited scope of the sample. In the present study, we have completed a statistically representative pilot experiment. Some improvements on the presentation speed of stimuli and the initial description of the tasks, were made for this study. All of them contributed to further facilitate the administration of the game by professionals with different background, or even to further facilitate self-administration as a screening tool. Even though, further research is needed to improve the resolution of the game for the identification of specific cognitive impairment conditions, as well as to achieve a complete assessment of the psychometric properties of the digital game, particularly, the psychometric reliability.

\section{CONCLUSIONS}

This study demonstrates the psychometric validity of the Episodix cognitive game. This is a clear step ahead in the introduction of serious games and machine learning for cognitive assessment, and therefore, for detecting MCI or Alzheimer's disease, that is, to predict cognitive impairment using data captured from serious games. After overcoming the limitations identified during a preliminary study, a new statistically-significant study was carried out. Best prediction results were obtained with the introduction of extremely randomized trees and boosting trees (i.e., ET and GB) as classification algorithms. However, more trials are needed to achieve normative data with clinical validity. Finally, as a future line of research, authors are exploring the introduction of item response theory (IRT) in Episodix, to address some the limitations of classical test theory (CTT), about psychometric properties.

\section{ACKNOWLEDGMENTS}

We will like to thank the support of the residence of Santa Marta of Vigo, the Association of Relatives of Alzheimer's Patients of Morrazo (AFAMO), and finally, and the Association of Relatives of Alzheimer's Patients and other Dementias of Galicia (AFAGA), to facilitate the selection of participants among its members for the present pilot study.

\section{References}

Aalbers T., Baars MAE., Rikkert MGMO., Kessels RPC. 2013. Puzzling With Online Games (BAM-COG): Reliability, Validity, and Feasibility of an Online Self-Monitor for Cognitive Performance in Aging Adults. Journal of medical Internet research 15. AERA., APA., NCME. 2014. The Standards for Educational and Psychological Testing. AERA.

Albert MS., DeKosky ST., Dickson D., Dubois B., Feldman HH., Fox NC., Gamst A., Holtzman DM., Jagust WJ., Petersen RC., Snyder PJ., Carrillo MC., Thies B., Phelps CH. 2011. The diagnosis of mild cognitive impairment due to Alzheimer's disease: recommendations from the National Institute on Aging-Alzheimer's Association workgroups on diagnostic guidelines for Alzheimer's disease. Alzheimer's \& dementia : the journal of the Alzheimer's Association 7:270-9. DOI: 10.1016/j.jalz.2011.03.008.

Banerjee S., Smith SC., Lamping DL., Harwood RH., Foley B., Smith P., Murray J., Prince M., Levin E., Mann A., others. 2006. Quality of life in dementia: more than just cognition. An analysis of associations with quality of life in dementia. Journal of Neurology, Neurosurgery \& Psychiatry 77:146-148.

Barlett JE., Kotrlik JW., Higgins CC. 2001. Organizational research: Determining appropriate sample size in survey research. Information technology, learning, and performance journal 19:43.

Batista GE., Monard MC. 2003. An analysis of four missing data treatment methods for supervised learning. Applied artificial intelligence 17:519-533.

Breiman L. 2001. Random forests. Machine learning 45:5-32.

Campos-Magdaleno M., Facal D., Lojo-Seoane C., Pereiro AX., Juncos-Rabadán O. 2017. Longitudinal Assessment of Verbal Learning and 
Memory in Amnestic Mild Cognitive Impairment: Practice Effects and Meaningful Changes. Frontiers in psychology 8:1231.

Chaytor N., Schmitter-Edgecombe M. 2003. The ecological validity of neuropsychological tests: A review of the literature on everyday cognitive skills. Neuropsychology review 13:181-197.

Chetelat G., Landeau B., Eustache F., Mezenge F., Viader F., De La Sayette V., Desgranges B., Baron J-C. 2005. Using voxel-based morphometry to map the structural changes associated with rapid conversion in MCI: a longitudinal MRI study. Neuroimage 27:934-946.

Cockrell JR., Folstein MF. 1987. Mini-Mental State Examination (MMSE). Psychopharmacology bulletin 24:689-692.

Cohen J. 1960. A coefficient of agreement for nominal scales. Educational and psychological measurement 20:37-46.

Cordell CB., Borson S., Boustani M., Chodosh J., Reuben D., Verghese J., Thies W., Fried LB., of Cognitive Impairment Workgroup MD., others. 2013. Alzheimer's Association recommendations for operationalizing the detection of cognitive impairment during the Medicare Annual Wellness Visit in a primary care setting. Alzheimer's \& Dementia 9:141-150.

Davis J., Goadrich M. 2006. The relationship between Precision-Recall and ROC curves. In: Proceedings of the 23rd international conference on Machine learning. 233-240.

F. B., P. N., S. L., M. H., M.-P. D., R. V. 2010. Multitasking and prospective memory: Can virtual reality be useful for diagnosis? Behavioural Neurology 23:209-211. DOI: 10.3233/BEN-2010-0297.

Facal D., Guàrdia-Olmos J., Juncos-Rabadán O. 2015. Diagnostic transitions in mild cognitive impairment by use of simple Markov models. International journal of geriatric psychiatry 30:669-676.

Farias ST., Harrell E., Neumann C., Houtz A. 2003. The relationship between neuropsychological performance and daily functioning in individuals with Alzheimer's disease: ecological validity of neuropsychological tests. Archives of Clinical Neuropsychology 18:655-672.

Freund Y., Schapire RE. 1997. A decision-theoretic generalization of on-line learning and an application to boosting. Journal of computer and system sciences 55:119-139.

Fukui Y., Yamashita T., Hishikawa N., Kurata T., Sato K., Omote Y., Kono S., Yunoki T., Kawahara Y., Hatanaka N., Tokuchi R., Deguchi K., Abe K. 2015. Computerized touch-panel screening tests for detecting mild cognitive impairment and Alzheimer's disease. Internal medicine (Tokyo, Japan) 54:895-902. DOI: 10.2169/internalmedicine.54.3931.

Garc \'〈ia-Laencina PJ., Sancho-Gómez J-L., Figueiras-Vidal AR. 2010. Pattern classification with missing data: a review. Neural Computing and Applications 19:263-282.

Geurts P., Ernst D., Wehenkel L. 2006. Extremely randomized trees. Machine learning 63:3-42.

Grambaite R., Selnes P., Reinvang I., Aarsland D., Hessen E., Gjerstad L., Fladby T. 2011. Executive dysfunction in mild cognitive impairment is associated with changes in frontal and cingulate white matter tracts. Journal of Alzheimer's Disease 27:453.

Hajian-Tilaki K. 2014. Sample size estimation in diagnostic test studies of biomedical informatics. Journal of biomedical informatics 48:193204.

Hawkins KA., Dean D., Pearlson GD. 2004. Alternative forms of the Rey Auditory Verbal Learning Test: a review. Behavioural neurology 15:99-107.

Hearst MA., Dumais ST., Osuna E., Platt J., Scholkopf B. 1998. Support vector machines. IEEE Intelligent Systems and their applications 13:1828.

Helkala E-L., Laulumaa V., Soininen H., Riekkinen PJ. 1989. Different error pattern of episodic and semantic memory in Alzheimer's disease and Parkinson's disease with dementia. Neuropsychologia 27:1241-1248.

Holtzman DM., Morris JC., Goate AM. 2011. Alzheimer's disease: the challenge of the second century. Science translational medicine 3:77sr1-$77 \mathrm{sr} 1$.

Howieson DB., Lezak MD. 2010. The neuropsychological evaluation. Essentials of Neuropsychiatry and Behavioral Neurosciences:29-46.

Iriarte Y., Diaz-Orueta U., Cueto E., Irazustabarrena P., Banterla F., Climent G. 2016. AULA-Advanced virtual reality tool for the assessment of attention: Normative study in Spain. Journal of Attention Disorders 20:542-568. DOI: http://dx.doi.org/10.1177/1087054712465335.

Juncos-Rabadán O., Facal D., Pereiro AX., Lojo-Seoane C. 2014. Visual memory profiling with CANTAB in mild cognitive impairment (MCI) subtypes. International journal of geriatric psychiatry 29:1040-1048.

Juncos-Rabadán O., Pereiro AX., Facal D., Rodriguez N., Lojo C., Caamaño JA., Sueiro J., Boveda J., Eiroa P. 2012. Prevalence and correlates of cognitive impairment in adults with subjective memory complaints in primary care centres. Dementia and geriatric cognitive disorders 33:226-232.

Kato PM., de Klerk S. 2017. Serious Games for Assessment: Welcome to the Jungle. Journal of Applied Testing Technology 18:1-6.

Kawahara Y., Ikeda Y., Deguchi K., Kurata T., Hishikawa N., Sato K., Kono S., Yunoki T., Omote Y., Yamashita T., Abe K. 2015. Simultaneous assessment of cognitive and affective functions in multiple system atrophy and cortical cerebellar atrophy in relation to computerized touch-panel screening tests. Journal of the Neurological Sciences 351:24-30. DOI: http://dx.doi.org/10.1016/j.jns.2015.02.010.

Knight RG., Titov N. 2009. Use of virtual reality tasks to assess prospective memory: Applicability and evidence. Brain impairment $10: 3-13$.

L. B., M. W., N.F. M., R. V., M. S., T. K., W. S. 2010. Evaluation of spatial processing in virtual reality using functional magnetic resonance imaging (fMRI). Cyberpsychology, Behavior, and Social Networking 13:211-215. DOI: 10.1089/cyber.2008.0343.

Landis JR., Koch GG. 1977. The measurement of observer agreement for categorical data. biometrics:159-174.

Lehmann C., Koenig T., Jelic V., Prichep L., John RE., Wahlund L-O., Dodge Y., Dierks T. 2007. Application and comparison of classification algorithms for recognition of Alzheimer's disease in electrical brain activity (EEG). Journal of neuroscience methods 161:342-350.

Lezak MD. 2004. Neuropsychological assessment. Oxford university press.

Libon DJ., Bondi MW., Price CC., Lamar M., Eppig J., Wambach DM., Nieves C., Delano-Wood L., Giovannetti T., Lippa C., others. 2011. Verbal serial list learning in mild cognitive impairment: A profile analysis of interference, forgetting, and errors. Journal of the International Neuropsychological Society 17:905-914.

Mario B., Massimiliano M., Chiara M., Alessandro S., Antonella C., Gianfranco F. 2009. White-coat effect among older patients with suspected cognitive impairment: prevalence and clinical implications. International journal of geriatric psychiatry 24:509-517.

Maroco J., Silva D., Rodrigues A., Guerreiro M., Santana I., de Mendonça A. 2011. Data mining methods in the prediction of Dementia: A realdata comparison of the accuracy, sensitivity and specificity of linear discriminant analysis, logistic regression, neural networks, support vector machines, classification trees and random forests. BMC research notes 4:299.

Nolin P., Banville F., Cloutier J., Allain P. 2013. Virtual Reality as a New Approach to Assess Cognitive Decline in the Elderly. Academic Journal of Interdisciplinary Studies 2:612-616. DOI: 10.5901/ajis.2013.v2n8p612.

Nolin P., Stipanicic A., Henry M., Lachapelle Y., Lussier-Desrochers D., Rizzo A “Skip”., Allain P. 2016. ClinicaVR: Classroom-CPT: A virtual reality tool for assessing attention and inhibition in children and adolescents. Computers in Human Behavior 59:327-333. DOI: http://dx.doi.org/10.1016/j.chb.2016.02.023. 
608

609

610

611

612

613

614

615

616

617

618

619

620

621

622

623

624

625

626

627

628

629

630

631

632

633

634

635

636

637

638

639

640

641

642

643

644

645

646

647

648

649

650

651

652

653

654

655

656

657

658

659
Nori R., Piccardi L., Migliori M., Guidazzoli A., Frasca F., De Luca D., Giusberti F. 2015. The virtual reality Walking Corsi Test. Computers in Human Behavior 48:72-77. DOI: http://dx.doi.org/10.1016/j.chb.2015.01.035.

Parsons TD., Larson P., Kratz K., Thiebaux M., Bluestein B., Buckwalter JG., Rizzo AA. 2004. Sex differences in mental rotation and spatial rotation in a virtual environment. Neuropsychologia 42:555-562.

Payan A., Montana G. 2015. Predicting Alzheimer's disease: a neuroimaging study with 3D convolutional neural networks. arXiv preprint arXiv:1502.02506.

Pedregosa F., Varoquaux G., Gramfort A., Michel V., Thirion B., Grisel O., Blondel M., Prettenhofer P., Weiss R., Dubourg V., others. 2011. Scikit-learn: Machine learning in Python. Journal of machine learning research 12:2825-2830.

Perry RJ., Watson P., Hodges JR. 2000. The nature and staging of attention dysfunction in early (minimal and mild) Alzheimer's disease: relationship to episodic and semantic memory impairment. Neuropsychologia 38:252-271.

Petersen RC. 2004. Mild cognitive impairment as a diagnostic entity. Journal of internal medicine 256:183-194.

Plancher G., Gyselinck V., Nicolas S., Piolino P., G. P., V. G., S. N., P. P. 2010. Age effect on components of episodic memory and feature binding: A virtual reality study. Neuropsychology 24:379-390. DOI: 10.1037/a0018680.

Plancher G., Tirard A., Gyselinck V., Nicolas S., Piolino P. 2012. Using virtual reality to characterize episodic memory profiles in amnestic mild cognitive impairment and Alzheimer's disease: Influence of active and passive encoding. Neuropsychologia 50:592-602.

Prince MJ. 2015. World Alzheimer Report 2015: the global impact of dementia: an analysis of prevalence, incidence, cost and trends. Alzheimer's Disease International.

Raspelli S., Pallavicini F., Carelli L., Morganti F., Poletti B., Corra B., Silani V., Riva G. 2011. Validation of a Neuro Virtual Reality-based version of the Multiple Errands Test for the assessment of executive functions. Studies in health technology and informatics 167:92-97.

Rasquin SMC., Lodder J., Visser PJ., Lousberg R., Verhey FRJ. 2005. Predictive accuracy of MCI subtypes for Alzheimer's disease and vascular dementia in subjects with mild cognitive impairment: a 2-year follow-up study. Dementia and geriatric cognitive disorders 19:113-119.

Robinson SJ., Brewer G. 2016. Performance on the traditional and the touch screen, tablet versions of the Corsi Block and the Tower of Hanoi tasks. Computers in Human Behavior 60:29-34. DOI: http://dx.doi.org/10.1016/j.chb.2016.02.047.

Souza AC de., Alexandre NMC., Guirardello E de B. 2017. Psychometric properties in instruments evaluation of reliability and validity. Epidemiologia e Serviços de Saúde 26:649-659.

Tarnanas I., Schlee W., Tsolaki M., Müri R., Mosimann U., Nef T. 2013. Ecological validity of virtual reality daily living activities screening for early dementia: longitudinal study. JMIR Serious Games 1:e1.

Tong T., Chignell M. 2014. Developing a serious game for cognitive assessment: choosing settings and measuring performance. Proceedings of the Second International Symposium of Chinese CHI.

Tripoliti EE., Fotiadis DI., Argyropoulou M., Manis G. 2010. A six stage approach for the diagnosis of the Alzheimer's disease based on fMRI data. Journal of biomedical informatics 43:307-320.

Unity. 2016.Scripting API Unity. Available at https://docs.unity3d.com/ScriptReference/

Valladares-Rodriguez S., Pérez-Rodr '’iguez R., Anido-Rifón L., Fernández-Iglesias M. 2016. Trends on the application of serious games to neuropsychological evaluation: A scoping review. Journal of Biomedical Informatics 64:296-319.

Valladares-Rodriguez S., Perez-Rodriguez R., Facal D., Fernandez-Iglesias MJ., Anido-Rifon L., Mouriño-Garcia M. 2017. Design process and preliminary psychometric study of a video game to detect cognitive impairment in senior adults. PeerJ 2017. DOI: 10.7717/peerj.3508.

Werner P., Rabinowitz S., Klinger E., Korczyn AD., Josman N. 2009. Use of the Virtual Action Planning Supermarket for the Diagnosis of Mild Cognitive Impairment. Dement Geriatr Cogn Disord 27:301-309.

Whitwell JL., Petersen RC., Negash S., Weigand SD., Kantarci K., Ivnik RJ., Knopman DS., Boeve BF., Smith GE., Jack CR. 2007. Patterns of atrophy differ among specific subtypes of mild cognitive impairment. Archives of Neurology 64:1130-1138.

WHO WHO., Others. 2015. First WHO ministerial conference on global action against dementia: meeting report, WHO Headquarters, Geneva, Switzerland, 16-17 March 2015.

Winblad B., Amouyel P., Andrieu S., Ballard C., Brayne C., Brodaty H., Cedazo-Minguez A., Dubois B., Edvardsson D., Feldman H., others. 2016. Defeating Alzheimer's disease and other dementias: a priority for European science and society. The Lancet Neurology 15:455-532. 


\section{Table $\mathbf{1}$ (on next page)}

Comparative of participants' cognitive assessment by cognitive group

MMSE cut-off score: 9-12 means AD is likely present. Spanish version of CVLT cut-off scores computed according to the results of Total Trial 1-5, Short Delay Free Recall, Long Delay Free Recall, age and years of education (Albert et al., 2011; Juncos-Rabadán et al., 2012; CamposMagdaleno et al., 2017). Memory complaints (by participants and informants) based on a 5point Likert scale: 1(never forget) to 5 (always forget). AD8, 0-1: normal cognition; 2 or greater: cognitive impairment is likely to be present. Finally, ADL: 0-20: total dependency; 21-60: severe dependency; 61-90: moderate dependency; 91-99: small dependency; and 100: autonomy. 
1 Table 1. Comparative of participants' cognitive assessment by cognitive group

\begin{tabular}{|l|c|c|c|}
\hline TESTS SCORES & $\mathrm{HC}$ & $\mathrm{MCl}$ & $\mathrm{AD}$ \\
\hline MMSE & $26.93 \pm 2.11$ & $25.37 \pm 2.55$ & $23.00 \pm 4.38$ \\
\hline $\begin{array}{l}\text { RI_AT: Total Trial 1-5 } \\
\text { [Spanish version of CVLT] }\end{array}$ & $47.25 \pm 5.83$ & $34.62 \pm 9.50$ & \\
\hline $\begin{array}{l}\text { RL_CP: Short Delay Free Recall } \\
\text { [Spanish version of CVLT] }\end{array}$ & $9.92 \pm 2.55$ & $6.31 \pm 1.74$ & \\
\hline $\begin{array}{l}\text { RL_LP: Long Delay Free Recall } \\
\text { [Spanish version of CVLT] }\end{array}$ & $10.93 \pm 2.39$ & $5.87 \pm 3.07$ & \\
\hline AD8 & $1.39 \pm 1.47$ & $1.75 \pm 1.18$ & $5.40 \pm 1.31$ \\
\hline ADL Barthel scale & $102.07 \pm 6.39$ & $100.00 \pm 7.28$ & $91.85 \pm 11.00$ \\
\hline Subjective memory complaints [by participants] & $1.99 \pm 0.51$ & $1.99 \pm 0.57$ & $2.47 \pm 0.51$ \\
\hline Subjective memory complaints [by relatives] & $1.82 \pm 0.73$ & $1.78 \pm 0.41$ & $2.46 \pm 0.64$ \\
\hline
\end{tabular}

2 Note: MMSE cut-off score: 9-12 means AD is likely present. Spanish version of CVLT cut-off scores computed according to the results of Total 3 Trial 1-5, Short Delay Free Recall, Long Delay Free Recall, age and years of education (Albert et al., 2011; Juncos-Rabadán et al., 2012;

4 Campos-Magdaleno et al., 2017). Memory complaints (by participants and informants) based on a 5-point Likert scale: 1(never forget) to 5

5 (always forget). AD8, 0-1: normal cognition; 2 or greater: cognitive impairment is likely to be present. Finally, ADL: 0-20: total dependency; 21-

6 60: severe dependency; 61-90: moderate dependency; 91-99: small dependency; and 100: autonomy. 


\section{Table 2 (on next page)}

Metrics about psychometric predicted validity of the Episodix game.

Episodix dataset (191 triples). ML algorithms: ET: Extra Trees classifier; GB: Gradient Boosting Classifier; AB: Ada Boost Classifier; SVM: Support Vector Machines; LR: Logistic regression; and RF: Random forest. Metrics: F1 score; Cohen's Kappa (i.e., used as index to order best classification); recall and precision, the last two distributed by cognitive group. Experiments were performances with $\mathrm{cv}$-fold cross validation ( $\mathrm{cv}=55)$ and default configuration in $\mathrm{ML}$ algorithms. 
2 Table 1. Metrics about psychometric predicted validity of the Episodix game.

\begin{tabular}{|c|c|c|c|c|c|c|c|c|}
\hline \multirow{2}{*}{$\begin{array}{l}\text { EPISODIX DATA SET } \\
\text { ML algorithms }\end{array}$} & \multirow[b]{2}{*}{$\mathrm{F} 1$} & \multirow[b]{2}{*}{$\mathrm{k} \downarrow$} & \multicolumn{3}{|c|}{ Recall } & \multicolumn{3}{|c|}{ Precision } \\
\hline & & & $\mathrm{HC}$ & $\mathrm{MCl}$ & $A D$ & $\mathrm{HC}$ & $\mathrm{MCl}$ & $A D$ \\
\hline GB: Gradient Boosting & 0,92 & 0,89 & 0,96 & 0,92 & 0,91 & 0,94 & 0,87 & 0,96 \\
\hline ET: Extra Trees & 0,91 & 0,88 & 0,91 & 0,94 & 0,95 & 0,97 & 0,76 & 0,96 \\
\hline RF: Random Forest & 0,89 & 0,86 & 0,86 & 1,00 & 0,95 & 0,99 & 0,66 & 0,96 \\
\hline AB: Ada Boost & 0,81 & 0,76 & 0,79 & 0,76 & 0,96 & 0,91 & 0,58 & 0,92 \\
\hline SVM: Support Vector Machine & 0,80 & 0,75 & 0,80 & 0,75 & 0,93 & 0,91 & 0,55 & 0,92 \\
\hline LR: Logistic Regression & 0,76 & 0,70 & 0,74 & 0,73 & 0,94 & 0,92 & 0,42 & 0,91 \\
\hline
\end{tabular}

3 Note: Episodix dataset (191 triples). ML algorithms: ET: Extra Trees classifier; GB: Gradient Boosting Classifier; AB: Ada Boost Classifier;

4 SVM: Support Vector Machines; LR: Logistic regression; and RF: Random forest. Metrics: F1 score; Cohen's Kappa (i.e., used as index to order 5 best classification); recall and precision, the last two distributed by cognitive group. Experiments were performances with cv-fold cross validation $6 \quad(\mathrm{cv}=55)$ and default configuration in ML algorithms. 


\section{Table 3 (on next page)}

Metrics about psychometric predicted validity of the Episodix, semantic memory and procedural memory games

Episodix, semantic memory and procedural memory dataset (458 triples). ML algorithms: GB: Gradient Boosting Classifier; ET: Extra Trees classifier; SVM: Support Vector Machines; LR: Logistic regression; AB: Ada Boost Classifier; and RF: Random forest. Metrics: F1 score; Cohen's Kappa (i.e., used as index to order best classification); recall and precision, the last two distributed by cognitive group. Experiments were performances with cv-fold cross validation ( $\mathrm{CV}=55)$ and default configuration in $\mathrm{ML}$ algorithms. 
2 Table 1. Metrics about psychometric predicted validity of the Episodix, semantic memory and procedural memory games.

\begin{tabular}{|l|c|c|c|c|c|c|c|}
\hline $\begin{array}{l}\text { EPISODIX+S+P } \\
\text { DATASET }\end{array}$ & & & \multicolumn{3}{|c|}{ Recall } & \multicolumn{2}{|c|}{ Precision } \\
\hline ML classifier & $\mathrm{F} 1$ & $\mathrm{k} \downarrow$ & $\mathrm{HC}$ & $\mathrm{MCl}$ & $\mathrm{AD}$ & $\mathrm{HC}$ & $\mathrm{MCI}$ \\
\hline ET: Extra Trees & 0,97 & 0,97 & 0,96 & 0,99 & 0,99 & 0,99 & 0,91 \\
\hline RF: Random Forest & 0,97 & 0,96 & 0,95 & 0,98 & 0,99 & 0,98 & 0,91 \\
\hline GB: Gradient Boosting & 0,96 & 0,95 & 0,95 & 0,94 & 0,98 & 0,96 & 0,93 \\
\hline SVM: Support Vector Machine & 0,91 & 0,90 & 0,92 & 0,82 & 0,99 & 0,90 & 0,90 \\
\hline LR: Logistic Regression & 0,84 & 0,81 & 0,77 & 0,80 & 0,98 & 0,90 & 0,98 \\
\hline AB: Ada Boost & 0,84 & 0,80 & 0,77 & 0,78 & 0,99 & 0,90 & 0,90 \\
\hline
\end{tabular}

3 Note: Episodix, semantic memory and procedural memory dataset (458 triples). ML algorithms: GB: Gradient Boosting Classifier; ET: Extra 4 Trees classifier; SVM: Support Vector Machines; LR: Logistic regression; AB: Ada Boost Classifier; and RF: Random forest. Metrics: F1 score; 5 Cohen's Kappa (i.e., used as index to order best classification); recall and precision, the last two distributed by cognitive group. Experiments 6 were performances with $\mathrm{cv}$-fold cross validation ( $\mathrm{cv}=55)$ and default configuration in ML algorithms. 
Figure 1

General subjects' characteristics according to cognitive group

Bars are distributed by cognitive groups: A. gender: men/women; B. age; C. chronic treatment: yes/no (i.e., 1/0); D. educational level, E. exercise level and F. socialize level, all of them based on a 5-point Likert scale: 1 (never) to 5 (always).
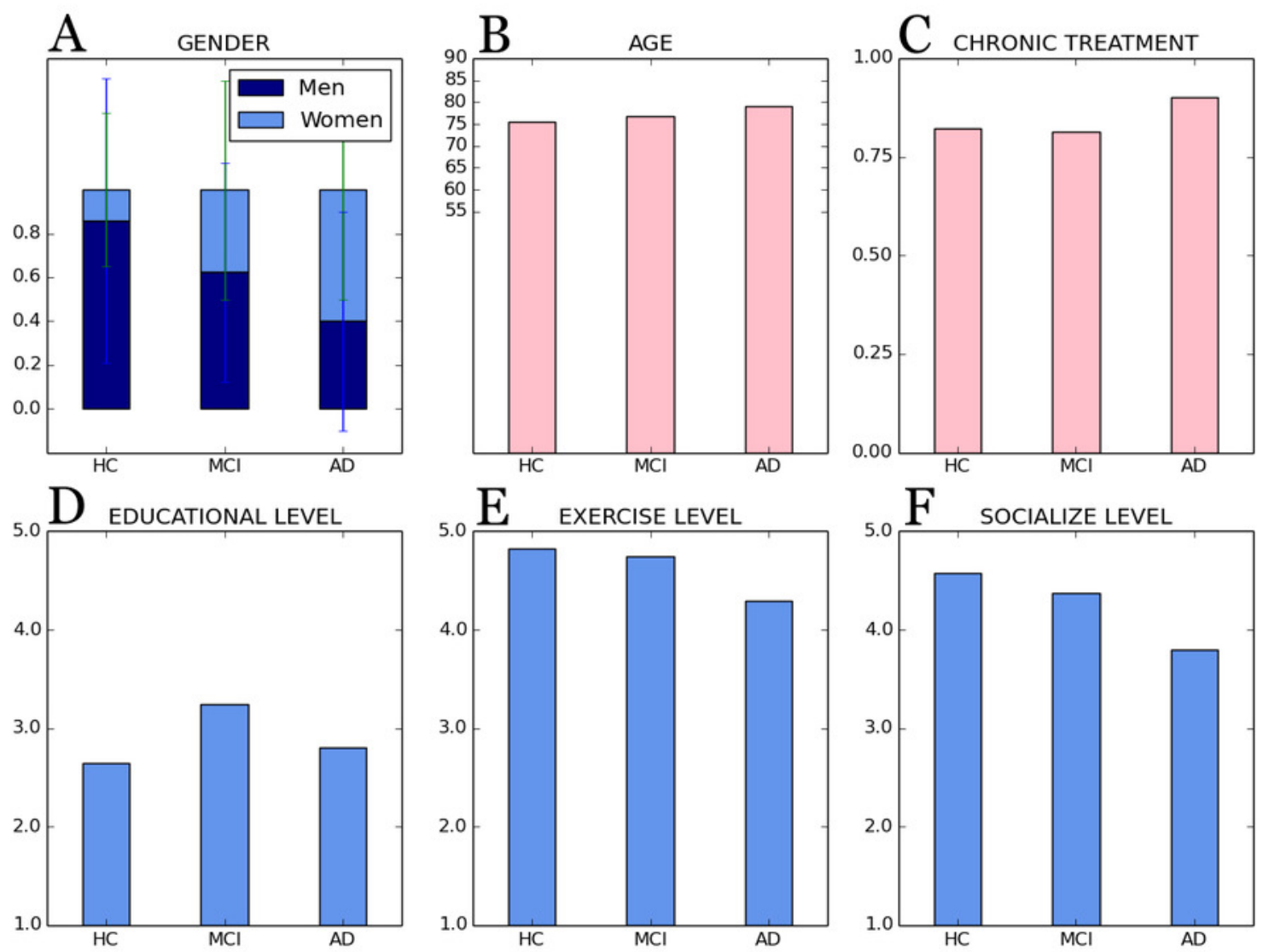


\section{Figure 2}

Some captures of cognitive game sessions

A and B: Episodix, C: semantic memory game and D: procedural memory game. Photo permission was required and granted in the patient informant sheet. Photo credit: the coauthor Sonia Valladares-Rodriguez.
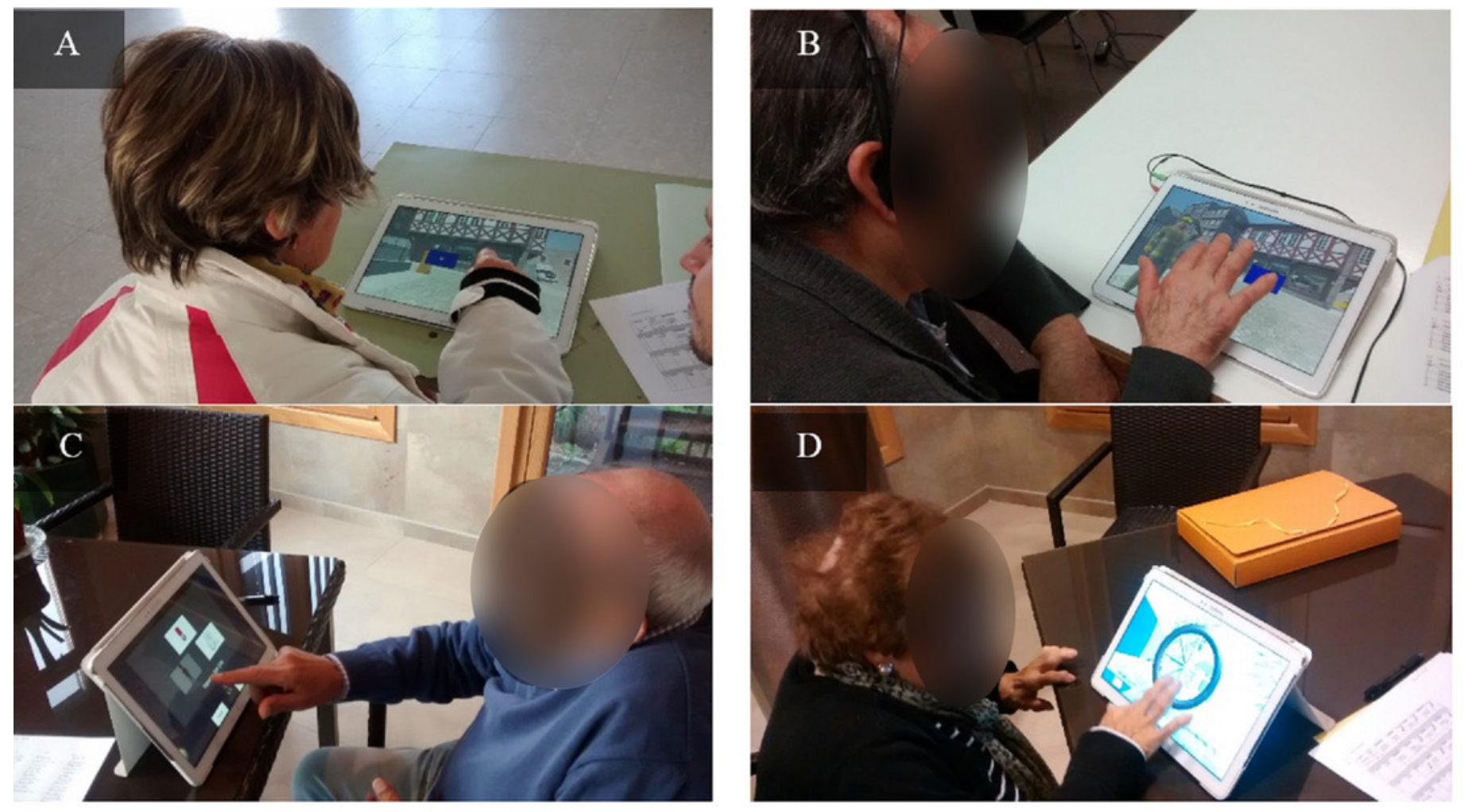
Figure 3

\section{Comparative of Precision-Recall curve metric to evaluate classifier output}

ML algorithms: A. [GB: Gradient Boosting Classifier]; B. [AB: Ada Boost Classifier]; C. [ET:

Extra Trees classifier]; D. [LR: Logistic regression]; E. [SVM: Support Vector Machines]; and F.

[RF: Random forest]. Metrics: precision-recall curve average for each cognitive group.

Experiments were performances with cv-fold cross validation ( $c v=55)$.
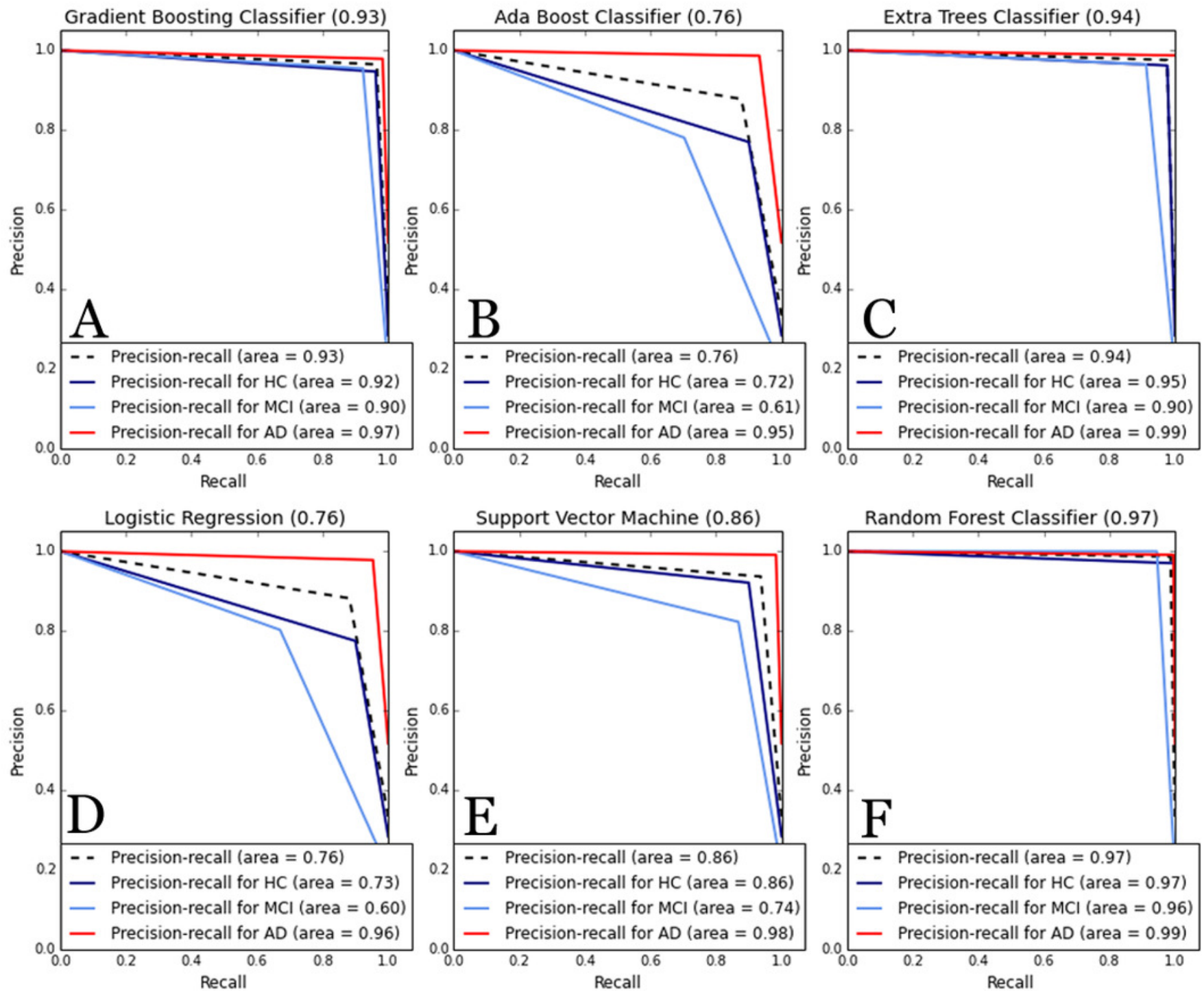
Figure 4

Comparative of Precision-Recall curve metric using the best performance algorithm (i.e. Gradient Boosting classifier).

A. [Episodix data set, GB: Gradient Boosting Classifier and chi-squared feature selection]; B. [Episodix data set, GB: Gradient Boosting Classifier and ANOVA F-test feature selection]; C. [Episodix data set, GB: Gradient Boosting Classifier and all features]; D. [Episodix+S+P data set, GB: Gradient Boosting Classifier and chi-squared feature selection]; E. [Episodix+S+P data set, GB: Gradient Boosting Classifier and ANOVA F-test feature selection]; and F. [Episodix+S+P data set, GB: Gradient Boosting Classifier and all features].

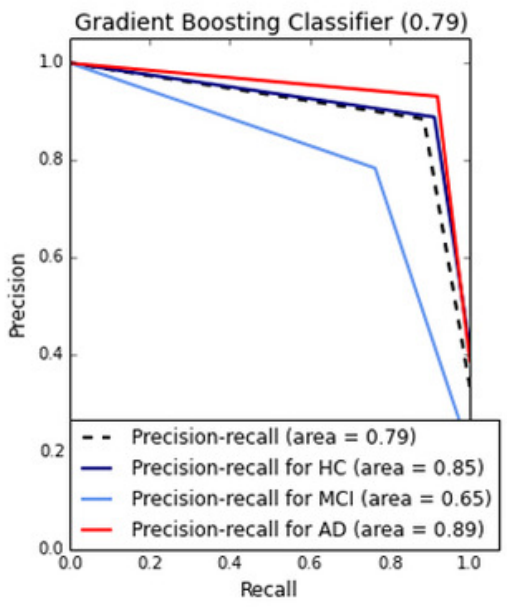

A. [Episodix]

(chi-squared feature selection)

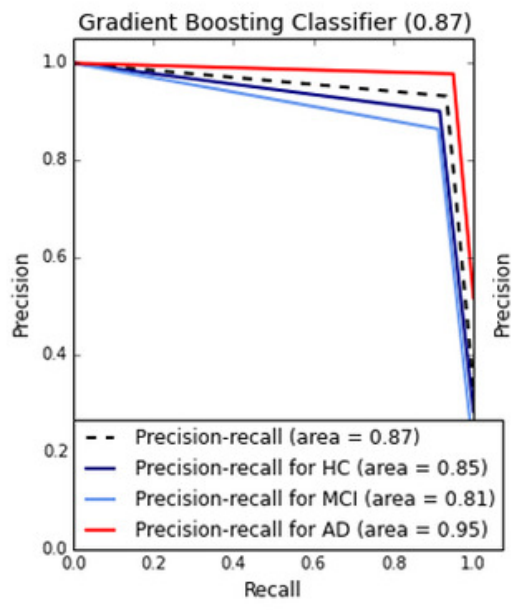

D. $[$ Episodix $+\mathrm{S}+\mathrm{P}]$

(chi-squared feature selection)
Peer] reviewing PDF | (2018:02:26202:1:1:NEW 13 Jul 2018)

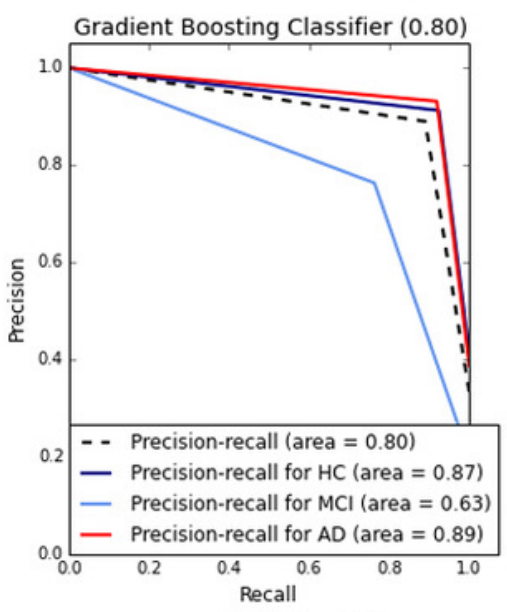

B. [Episodix]

(ANOVA feature selection)

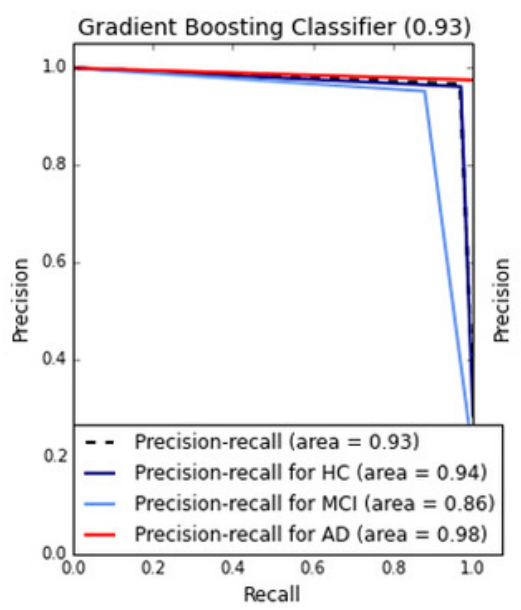

E. [Episodix $+\mathrm{S}+\mathrm{P}]$ (ANOVA feature selection)
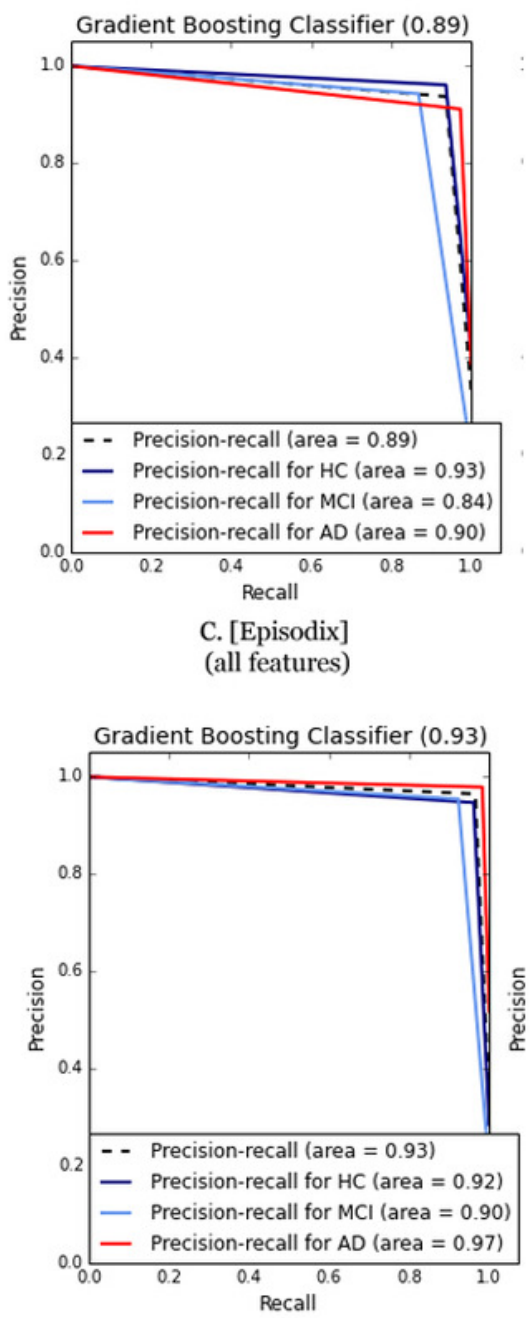

F. $[$ Episodix $+\mathrm{S}+\mathrm{P}]$ (all features) 


\section{Table 4 (on next page)}

Appendix A. List of 10-features most informative of the Episodix digital game

List of 10 -features most informative of the Episodix digital game, usign chi-squared and ANOVA F-test 
1 Appendix A. List of 10-features most informative of the Episodix digital game

2

3 Appendix Table 1. List of 10-features most informative of the Episodix digital game.

\begin{tabular}{|c|c|c|c|}
\hline \multicolumn{4}{|l|}{ EPISODIX } \\
\hline \multirow{9}{*}{\multicolumn{2}{|c|}{ Chi2 }} & & 1. RCL-LP2_Omissions: \\
\hline & & & 2. RCL-LP1_Omissions \\
\hline & & & 3. RI-B1_Failures \\
\hline & & & 4. Rec-LP_Repetitions \\
\hline & & & 5. RCL-CP1_Omissions \\
\hline & & & 6. RL-LP_Failures \\
\hline & & & 7. RI-A1_Failures \\
\hline & & & 8. RL-CP_Failures \\
\hline & & & 9. RI-A2 Failures \\
\hline \multirow{10}{*}{\multicolumn{2}{|c|}{ ANOVA F-value }} & & 1. RI-A3_Omissions \\
\hline & & & 2. RL-LP_Guesses \\
\hline & & & 3. RL-LP_Omissions \\
\hline & & & 4. Rec-LP_Repetitions \\
\hline & & & 5. RL-CP_Failures \\
\hline & & & 6. RI-A3_Failures \\
\hline & & & 7. RL-LP_Failures \\
\hline & & & 8. RI-A1_Failures \\
\hline & & & 9. RI-B1_Failures \\
\hline & & & 10. RI-A2_Failures' \\
\hline \multicolumn{4}{|c|}{ EPISODIX + semantic memory and procedural memory game } \\
\hline \multirow{10}{*}{\multicolumn{2}{|c|}{ Chi2 }} & & 1. RCL-CP1_Omissions \\
\hline & & & 2. RL-CP_Failures \\
\hline & & & 3. RI-A3_Failures \\
\hline & & & 4. Rec-LP_Repetitions \\
\hline & & & 5. RI-A2_Failures \\
\hline & & & 6. procedurixTimeDuration \\
\hline & & & 7. RL-LP_Failures \\
\hline & & & 8. 8. RI-A1_Failures \\
\hline & & & 9. RI-B1_Failures \\
\hline & & & 10. RCL-LP1_Omissions \\
\hline \multirow{10}{*}{\multicolumn{2}{|c|}{ ANOVA F-value }} & & 1.RL-LP_Guesses \\
\hline & & & 2.RL-LP_Omissions \\
\hline & & & 3.RL-CP_Failures \\
\hline & & & 4.RL-LP_Failures \\
\hline & & & 5.RI-B1_Failures \\
\hline & & & 6.RI-A1_Failures \\
\hline & & & 7.procedurixTimeDuration \\
\hline & & & 8.RI-A2_Failures \\
\hline & & & 9.Rec-LP_Repetitions \\
\hline & & & 10.RI-A3_Failures \\
\hline
\end{tabular}




\section{Table 5(on next page)}

\section{Appendix B. Metrics about psychometric study}

Appendix B Table 1 Episodix dataset (191 triples). Features: 10 most informative features, age and genre, using chi-squared and ANOVA F-value methods. ML algorithms: ET: Extra Trees classifier; GB: Gradient Boosting Classifier; AB: Ada Boost Classifier; SVM: Support Vector Machines; LR: Logistic regression; and RF: Random forest. Metrics: F1 score; Cohen's Kappa (i.e., used as index to order best classification); average and precision-recall area distributed by cognitive group. Cross validation (cv-fold $=55$ ).

Appendix B Table 2 Episodix, semantic memory and procedural memory dataset (458 triples). Features: 10 most informative features, age and genre, using chi-squared and ANOVA F-value methods. ML algorithms: ET: Extra Trees classifier; GB: Gradient Boosting Classifier; AB: Ada Boost Classifier; SVM: Support Vector Machines; LR: Logistic regression; and RF: Random forest. Metrics: F1 score; Cohen's Kappa (i.e., used as index to order best classification); average and precision-recall area distributed by cognitive group. Cross validation ( $\mathrm{cv}$-fold $=55)$.

Appendix B Table 3 Episodix dataset (191 triples) and Episodix, semantic memory and procedural memory dataset (458 triples). All Features (e.g., 81 features for EPISODIX and 100 features for EPISODIX+S+P). ML algorithms: ET: Extra Trees classifier; GB: Gradient Boosting Classifier; AB: Ada Boost Classifier; SVM: Support Vector Machines; LR: Logistic regression; and RF: Random forest. Metrics: F1 score; Cohen's Kappa (i.e., used as index to order best classification); average and precision-recall area distributed by cognitive group. Cross validation (cv-fold $=55$ ). 


\section{Appendix B. Metrics about psychometric study}

2 Appendix B Table 1. Metrics about psychometric predicted validity using the most informative features of EPISODIX dataset, sorted by Cohen's kappa coefficient.

\section{EPISODIX DATA SET}

GB: GRADIENT BOOSTING
ET: EXTRA TREES
RF: RANDOM FOREST
SVM: SUPPORT VECTOR MACHINE
AB: ADA BOOST
LR: LOGISTIC REGRESSION

\section{CHI-SQUARE}

ANOVA-F

\begin{tabular}{|c|c|c|c|c|c|c|c|c|c|c|c|}
\hline \multirow[b]{2}{*}{$\mathrm{F} 1$} & \multicolumn{5}{|c|}{ Precision-Recall area } & \multicolumn{6}{|c|}{ Precision-Recall area } \\
\hline & $k \downarrow$ & $\mathrm{HC}$ & $\mathrm{MCl}$ & $A D$ & ALL & F1 & $\mathrm{k} \downarrow$ & $\mathrm{HC}$ & $\mathrm{MCl}$ & $A D$ & ALL \\
\hline 0,87 & 0,82 & 0,85 & 0,65 & 0,89 & 0,79 & 0,87 & 0,83 & 0,87 & 0,63 & 0,89 & 0,8 \\
\hline 0,86 & 0,81 & 0,85 & 0,66 & 0,86 & 0,79 & 0,84 & 0,79 & 0,83 & 0,6 & 0,86 & 0,76 \\
\hline 0,85 & 0,80 & 0,84 & 0,61 & 0,87 & 0,78 & 0,83 & 0,76 & 0,81 & 0,6 & 0,83 & 0,75 \\
\hline 0,68 & 0,59 & 0,64 & 0,32 & 0,8 & 0,59 & 0,71 & 0,64 & 0,67 & 0,36 & 0,86 & 0,63 \\
\hline 0,69 & 0,58 & 0,63 & 0,4 & 0,77 & 0,6 & 0,67 & 0,54 & 0,7 & 0,32 & 0,73 & 0,58 \\
\hline 0,59 & 0,52 & 0,63 & 0,22 & 0,76 & 0,54 & 0,62 & 0,56 & 0,64 & 0,24 & 0,81 & 0,56 \\
\hline
\end{tabular}
Boosting Classifier; AB: Ada Boost Classifier; SVM: Support Vector Machines; LR: Logistic regression; and RF: Random forest. Metrics: F1 score; Cohen's Kappa (i.e., used as index to order best classification); average and precision-recall area distributed by cognitive group. Cross validation (cv-fold $=55$ ).

Appendix B Table 2. Metrics about psychometric predicted validity using the most informative features of EPISODIX+S+P dataset, sorted by Cohen's kappa coefficient

\begin{tabular}{|c|c|c|c|c|c|c|c|c|c|c|c|c|}
\hline \multirow[t]{3}{*}{ EPISODIX+S+P DATA SET } & \multicolumn{6}{|c|}{ CHI-SQUARE } & \multicolumn{6}{|c|}{ ANOVA-F } \\
\hline & \multirow[b]{2}{*}{$\mathrm{F} 1$} & \multicolumn{5}{|c|}{ Precision-Recall area } & & & \multicolumn{4}{|c|}{ Precision-Recall area } \\
\hline & & $k \downarrow$ & $\mathrm{HC}$ & $\mathrm{MCl}$ & $A D$ & ALL & F1 & $\mathrm{k} \downarrow$ & $\mathrm{HC}$ & $\mathrm{MCl}$ & $A D$ & ALL \\
\hline GB: GRADIENT BOOSTING & 0,92 & 0,89 & 0,85 & 0,81 & 0,95 & 0,87 & 0,96 & 0,95 & 0,94 & 0,86 & 0,98 & 0,93 \\
\hline ET: EXTRA TREES & 0,88 & 0,83 & 0,76 & 0,75 & 0,92 & 0,81 & 0,93 & 0,90 & 0,89 & 0,79 & 0,95 & 0,88 \\
\hline RF: RANDOM FOREST & 0,88 & 0,82 & 0,75 & 0,76 & 0,91 & 0,81 & 0,93 & 0,91 & 0,9 & 0,8 & 0,95 & 0,88 \\
\hline SVM: SUPPORT VECTOR MACHINE & 0,71 & 0,60 & 0,56 & 0,46 & 0,81 & 0,61 & 0,74 & 0,65 & 0,56 & 0,49 & 0,87 & 0,64 \\
\hline AB: ADA BOOST & 0,68 & 0,58 & 0,56 & 0,38 & 0,8 & 0,58 & 0,69 & 0,60 & 0,54 & 0,42 & 0,81 & 0,59 \\
\hline LR: LOGISTIC REGRESSION & 0,61 & 0,54 & 0,5 & 0,29 & 0,84 & 0,55 & 0,59 & 0,50 & 0,58 & 0,21 & 0,83 & 0,54 \\
\hline
\end{tabular}

8 Note: Episodix, semantic memory and procedural memory dataset (458 triples). Features: 10 most informative features, age and genre, using chi-squared and ANOVA F-value methods. ML algorithms:

9 ET: Extra Trees classifier; GB: Gradient Boosting Classifier; AB: Ada Boost Classifier; SVM: Support Vector Machines; LR: Logistic regression; and RF: Random forest. Metrics: F1 score; Cohen's

10 Kappa (i.e., used as index to order best classification); average and precision-recall area distributed by cognitive group. Cross validation (cv-fold $=55$ ). 
Appendix B Table 3. Metrics about psychometric predicted validity using the all features of datasets, sorted by Cohen's kappa coefficient.

EPISODIX DATA SET

EPISODIX+S+P DATA SET

\begin{tabular}{|c|c|c|c|c|c|c|c|c|c|c|c|c|}
\hline & \multirow[b]{2}{*}{$\mathrm{F} 1$} & \multicolumn{5}{|c|}{ Precision-Recall area } & \multirow[b]{2}{*}{$\mathrm{F} 1$} & \multirow[b]{2}{*}{$\mathrm{k} \downarrow$} & \multicolumn{4}{|c|}{ Precision-Recall area } \\
\hline & & $\mathrm{k} \downarrow$ & $\mathrm{HC}$ & $\mathrm{MCl}$ & $A D$ & ALL & & & $\mathrm{HC}$ & $\mathrm{MCl}$ & $A D$ & ALL \\
\hline GB: GRADIENT BOOSTING & 0,93 & 0,90 & 0,93 & 0,84 & 0,9 & 0,89 & 0,96 & 0,94 & 0,92 & 0,9 & 0,97 & 0,93 \\
\hline ET: EXTRA TREES & 0,92 & 0,90 & 0,92 & 0,78 & 0,93 & 0,88 & 0,97 & 0,97 & 0,96 & 0,92 & 0,99 & 0,95 \\
\hline RF: RANDOM FOREST & 0,91 & 0,88 & 0,88 & 0,76 & 0,93 & 0,86 & 0,98 & 0,97 & 0,97 & 0,93 & 0,99 & 0,96 \\
\hline AB: ADA BOOST & 0,81 & 0,76 & 0,76 & 0,52 & 0,91 & 0,73 & 0,84 & 0,80 & 0,72 & 0,61 & 0,95 & 0,76 \\
\hline SVM: SUPPORT VECTOR MACHINE & 0,80 & 0,75 & 0,77 & 0,5 & 0,89 & 0,72 & 0,91 & 0,90 & 0,86 & 0,74 & 0,98 & 0,86 \\
\hline LR: LOGISTIC REGRESSION & 0,76 & 0,70 & 0,72 & 0,42 & 0,89 & 0,68 & 0,84 & 0,81 & 0,73 & 0,6 & 0,96 & 0,76 \\
\hline
\end{tabular}

Note: Episodix dataset (191 triples) and Episodix, semantic memory and procedural memory dataset (458 triples). All Features (e.g., 81 features for EPISODIX and 100 features for EPISODIX+S+P). score; Cohen's Kappa (i.e., used as index to order best classification); average and precision-recall area distributed by cognitive group. Cross validation $(\mathrm{cv}-\mathrm{fold}=55$ ). 


\section{Table 6(on next page)}

\section{Appendix C. Screenshots of serious games}

Fig. 1 Screenshots of Episodix serious game, during yes/no recognition phase

Fig. 2 Screenshots of semantic memory game, during the initial training phase

Fig. 3 Screenshots of procedural memory game, after the $1^{\text {st }}$ execution and during the $3^{\text {th }}$ one, where ball's size varies. 


\section{Appendix C. Screenshots of serious games}

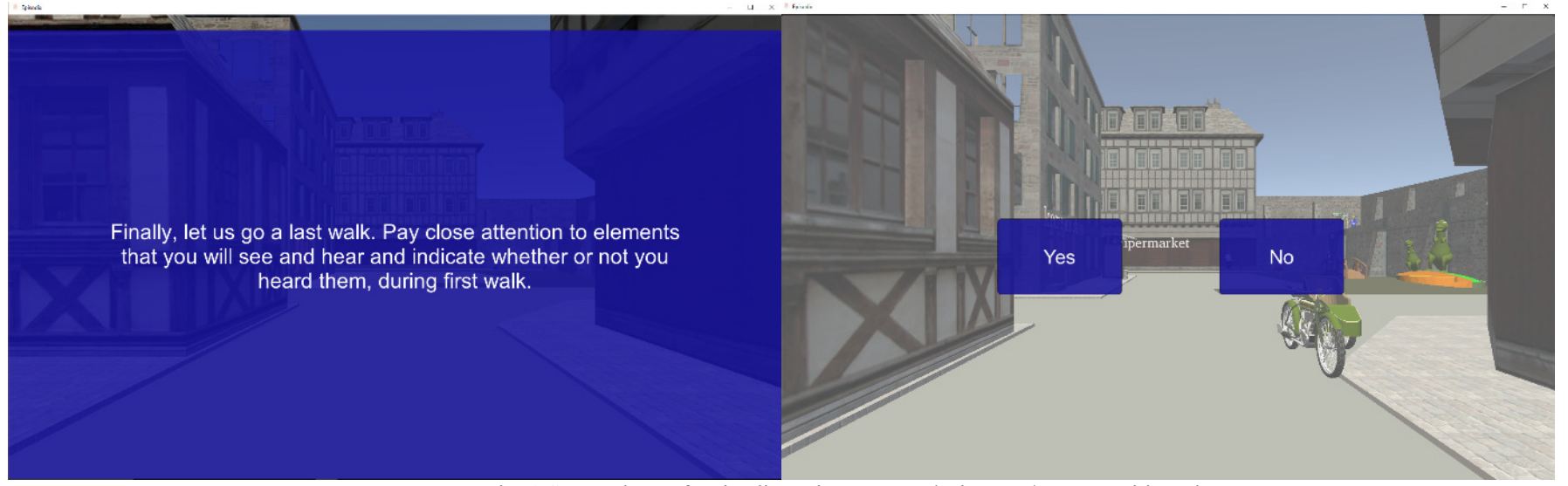

Fig. 1 Screenshots of Episodix serious game, during yes/no recognition phase

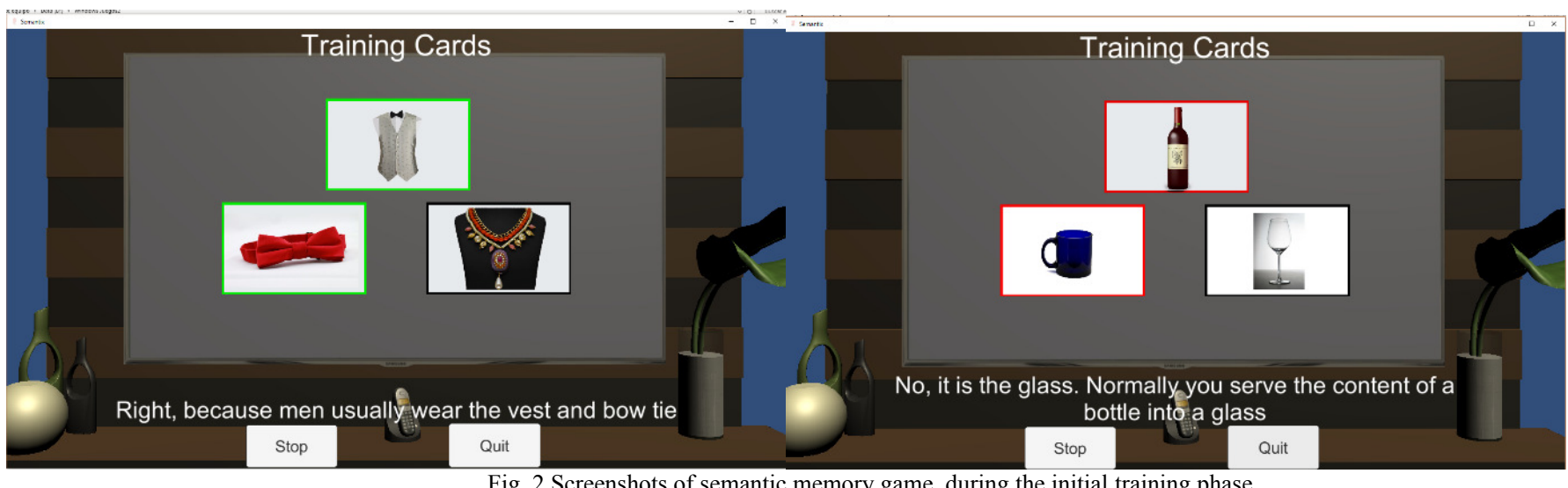

Fig. 2 Screenshots of semantic memory game, during the initial training phase 


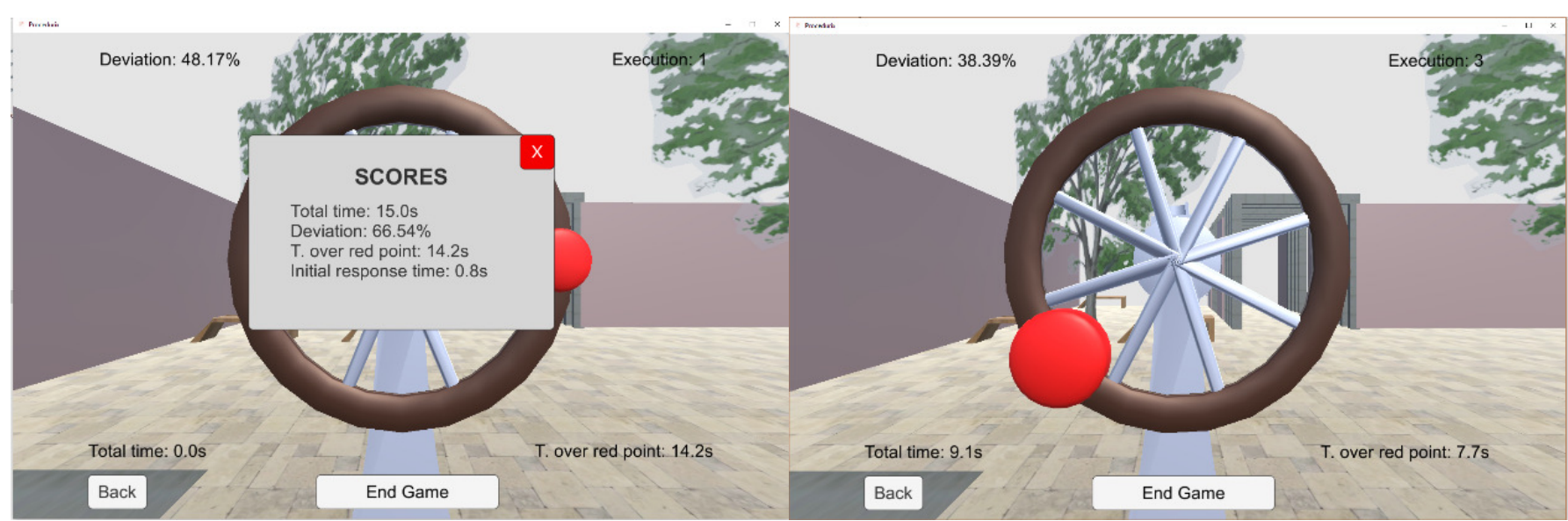

Fig. 3 Screenshots of procedural memory game, after the $1^{\text {st }}$ execution and during the $3^{\text {th }}$ one, where ball's size varies. 